\title{
An Ensemble of Cooperative Extended Kohonen Maps for Complex Robot Motion Tasks ${ }^{1}$
}

\author{
Kian Hsiang Low \\ bryanlow@cs.cmu.edu \\ Department of Electrical and Computer Engineering, Carnegie Mellon University, 5000 Forbes \\ Avenue, Pittsburgh, PA 15213-3890, USA \\ Wee Kheng Leow \\ leowwk@comp.nus.edu.sg \\ Department of Computer Science, National University of Singapore, 3 Science Drive 2, Singa- \\ pore 117543, Singapore \\ Marcelo H. Ang, Jr. \\ mpeangh@nus.edu.sg \\ Department of Mechanical Engineering, National University of Singapore, 10 Kent Ridge \\ Crescent, Singapore 119260, Singapore
}

Self-organizing feature maps such as Extended Kohonen Maps (EKMs) have been very successful at learning sensorimotor control for mobile robot tasks. This paper presents a new ensemble approach called cooperative EKMs with indirect mapping to achieve complex robot motion. An indirect-mapping EKM self-organizes to map from the sensory input space to the motor control space indirectly via a control parameter space. Quantitative evaluation reveals that indirect mapping can provide finer, smoother, and more efficient motion control than does direct mapping by operating in a continuous, rather than discrete, motor control space. It is also shown to outperform basis function neural networks. Furthermore, training its control parameters with recursive least squares enables faster convergence and better performance compared to gradient descent. The cooperation and competition of multiple self-organized EKMs allow a non-holonomic mobile robot to negotiate unforeseen, concave, closely spaced, and dynamic obstacles. Qualitative and quantitative comparisons with neural network ensembles employing weighted sum reveal that our method can achieve more sophisticated

\footnotetext{
${ }^{1}$ The final version of this article has been published in Neural Computation, Vol. 17, Issue 6, pages 1411-1445, Jun 2005, published by The MIT Press.
} 
motion tasks even though the weighted-sum ensemble approach also operates in continuous motor control space.

\section{Introduction}

Goal-directed, collision-free motion in a complex, dynamic, and unpredictable environment is an important task for an autonomous mobile robot operating alone or in a team. In particular, this task is widely employed in service and field robotics (Shastri, 1999), which includes sewer inspection (Hertzberg et al., 1998), cleaning and housekeeping (Fiorini et al., 2000), surveillance (Rybski et al., 2002), sensor network coverage (Howard et al., 2002), search and rescue (Davids, 2002), and tour guides (Burgard et al., 1999). All these applications require the mobile robot to perform target or goal reaching movements while avoiding undesirable and potentially dangerous impact with obstacles or other robots in its team. The robot motion control problem can be stated succinctly as follows: Given an initial state described by the sensory input vector $\mathbf{u}(0)$ in the sensory input space $\mathcal{U}$, determine a collision-free sequence of motor control vectors $\mathbf{c}(t), t=0, \ldots, T-1$, in the motor control space $\mathcal{C}$ that moves the robot towards a desired goal state described by $\mathbf{u}(T) \in \mathcal{U}$.

Three general classes of algorithms have been investigated for learning sensorimotor control, which is required for the above task: multivariate regression, reinforcement learning, and feature mapping. The first approach formulates the problem as a nonlinear multivariate regression problem and trains a multilayer perceptron (MLP) to perform continuous mapping from $\mathcal{U}$ to $\mathcal{C}$ (Pomerleau, 1991; Sharkey, 1998; Tani and Fukumura, 1994). It offers good generalization capability. However, prior to training the network, training samples have to be collected for every time step $t=0, \ldots, T-1$ to define the quantitative error signals. This sample collection process can be very difficult and tedious, if not impossible, for a mobile robot.

The reinforcement learning approach (Kaelbling et al., 1996; Sutton, 1998) circumvents the above difficulty by providing a qualitative success or failure feedback only at the end of executing the motor control sequence. It estimates how well each previously executed motor control vector $\mathbf{c}(t)$ contributes to the overall success or failure of achieving the desired goal and modifies the algorithm accordingly. The training process tends to converge slowly due to sparse reinforcements and imprecise estimate of each motor control vector's contribution.

The third approach uses a Self-Organizing Feature Map (SOFM) (Kohonen, 
2000) such as the Extended Kohonen Map (EKM) (Ritter and Schulten, 1986) that self-organizes to partition the continuous input (or output) space into localized regions. The generalization capability of the feature map arises from its self-organization during training such that each neuron is trained to map a localized sensory region to a desired motor control output. As compared to predefined, uniform partitioning of the feature space (Kuperstein, 1991; Schaal and Atkeson, 1998; Zalama et al., 1995), self-organization may lead to better performance and learning efficiency because more neural resources are automatically allocated to frequently encountered sensory regions during learning (Martinetz et al., 1990; Santamária et al., 1998). This approach increases the resolution of the sensory representation in the frequently encountered regions. Such a behavior is reminiscent of biological sensorimotor systems where frequently practiced movements become more fluid and accurate.

This paper describes a new feature map approach to learning sensorimotor control called cooperative EKMs with indirect mapping. An indirect-mapping EKM (Low et al., 2002) differs from existing direct-mapping methods (Cameron et al., 1998; Heikkonen and Koikkalainen, 1997; Rao and Fuentes, 1998; Ritter and Schulten, 1986; Smith, 2002; Touzet, 1997; Versino and Gambardella, 1995) in two ways:

1. Direct-mapping methods map a sensory input directly to a motor control command. In contrast, our indirect-mapping approach maps a sensory input indirectly to a motor control command through control parameters.

2. As a consequence, the indirect-mapping approach maps continuous sensory input space to continuous motor control space (see Section 3.1 for detailed discussion). On the other hand, direct-mapping methods map continuous sensory input space to discrete motor control commands.

The motor control space is often discretized into a set of commands to be used by reinforcement learning algorithms (Millán et al., 2002; Santamária et al., 1998; Smith, 2002; Touzet, 1997), committee machines with voting schemes (Battiti and Colla, 1994; Hansen and Salamon, 1990; Kittler et al., 1998; Sharkey and Sharkey, 1997), and robot action selection mechanisms (Decugis and Ferber, 1998; Huntsberger and Rose, 1998; Maes, 1995; Rosenblatt, 1997). However, recent autonomous agent research in dynamical systems theory (Beer, 1995; Port and van Gelder, 1995) and reinforcement learning (Millán et al., 2002; Smart and Kaelbling, 2000) advocates operating in continuous motor control space, which enables our indirect-mapping method to provide finer, smoother, and more efficient motion control than does direct mapping (Section 4.1). Such a 
high degree of smoothness, flexibility, and precision in motion control is essential for efficiently executing complex tasks and interacting with humans.

It is well understood how a SOFM or EKM is used for learning sensorimotor control (Littmann and Ritter, 1996; Walter and Schulten, 1993). However, the non-trivial problem of combining multiple SOFMs or EKMs for sophisticated control (e.g., negotiation of unforeseen complex obstacles and cooperative multi-robot tracking of moving targets (Low et al., 2003)) is not well studied. If solved poorly, the control outputs produced while performing a complex motion task may be unexpected or undesirable. For example, a widely used ensemble technique for motion control that combines neural network outputs via weighted sum (e.g., ensemble averaging and mixture of experts) (Hashem, 1997; Haykin, 1999; Jacobs, 1995) causes the robot to be trapped easily by unforeseen, complex obstacles. This navigation issue is central to the robotics community as it is often encountered during robot motion in a realworld environment (Kim and Khosla, 1992; Koren and Borenstein, 1991; Rimon and Koditschek, 1992). Note that such a problem will arise even when SOFMs or EKMs are utilized in the weighted-sum ensemble (Section 4.2).

To solve this robot motion problem, we propose a new ensemble approach called cooperative EKMs. The cooperation and competition of multiple EKMs (Low et al., 2003) that self-organize in the same manner can enable a nonholonomic mobile robot to negotiate unforeseen, concave, and closely spaced obstacles (Section 4.2). In contrast, a robot controlled by weighted-sum ensemble (Low et al., 2002) may fail in such tasks even though the networks also use continuous motor control space (Section 4.2). Before proceeding into the details of cooperative EKMs, we will discuss some related work first.

\section{Related Work}

In a MLP, all the training data are used to fit a single global model or representation. Therefore, during learning, all the network weights are susceptible to negative interference that may arise due to dynamically changing data distributions (Schaal and Atkeson, 1998). On the other hand, an EKM fits localized regions of data, rather than the entire region of interest, into local models or representations, thus localizing the effects of interference. Consequently, learning of a single new training data affects fewer network weights in an EKM than in a MLP (Atkeson et al., 1997; Martinetz et al., 1990). The cost of training in an EKM is kept small by imposing a topology among the neurons such that each learning step involves a subset of neighboring neurons. Initially, the 
subsets are chosen large, resulting in rapid learning of the coarse sensorimotor mapping. As learning progresses, the size of the subsets is gradually reduced to refine the mapping more and more locally. This strategy allows computationally efficient and accurate training of many neurons and facilitates scaling up the EKM to a larger number of neurons for further improved accuracy. An EKM also uses a smaller proportion of network weights for motor control prediction and has been reported to achieve more precise robot positioning than a MLP (Gorinevsky and Connolly, 1994; Jansen et al., 1995; van der Smagt et al., 1994). However, like other local model networks, an EKM suffers from the "curse of dimensionality". That is, the proportion of training data lying within a fixed-radius neighborhood of a point decreases exponentially with increasing number of dimensions of the input space.

Basis function network (BFN) is another type of local model network like EKM. However, it is architecturally different from EKM in that each incoming sensory input is reduced to activation strengths by basis functions, which are in turn linearly mapped to a corresponding control output. To do so, the output weights of all BFN neurons are required in predicting the target reaching motion. In contrast, the EKM uses only the winning neuron's output weights to map each sensory input to a control output (Section 3.1). During network learning, BFN updates all its output weights with each training data. On the other hand, only the output weights of the winning neuron and its neighbors in the EKM are updated (Section 3.5). As such, BFN may experience much more interference during online learning than an EKM.

Our indirect-mapping EKM resembles the EKM models of (Littmann and Ritter, 1996; Walter and Schulten, 1993), which utilize locally linear mappings. In their models, each neuron stores both the motor control vector and the matrix of motor control parameters as output weights (Eq. 1). On the other hand, each neuron in the indirect-mapping EKM only stores the matrix of motor control parameters. In the context of our paper, their EKM models and indirectmapping EKM, respectively, use 1800 and 1350 parameters in a network of $15 \times 15$ neurons (Table 1 ). The extra parameters employed by their models are not necessary in achieving good target reaching performance, as demonstrated by the indirect-mapping EKM in this paper (Section 4.1). Furthermore, we have shown that training the control parameters with recursive least squares enables faster convergence and better performance compared to gradient descent. Their EKM models (Littmann and Ritter, 1996; Walter and Schulten, 1993) have only used gradient descent to learn the control parameters.

It is typical for a robot to require a very large number of training data for accurate sensorimotor learning. The collection of these data for offline training 
is a very difficult, tedious, if not impossible, task. Therefore, online learning is preferred over offline to eliminate the need of storing these data and avoid running complex batch training algorithms such as support vector machines (Schaal et al., 2002). Hence, our focus in this paper will be on online learning.

Previously, Walter and Ritter (1996) have proposed an ensemble of multiple SOFMs called hierarchical PSOM (Parameterized Self-Organizing Maps) for learning sensorimotor control of robot arm under different system contexts. PSOM is a variant of SOFM that can learn with a small set of training data and still achieve good accuracy. To be able to do so, PSOM performs interpolation on a continuous mapping manifold using a small set of predefined, dataindependent basis functions, and weight vectors constructed directly from the data samples. To ensure good interpolation, the data samples have to be topologically ordered to form the weight vectors. Furthermore, the minimization of the distance function in SOFM algorithm (Eq. 2) turns into a continuous search problem for PSOM due to its continuous manifold. For hierarchical PSOM, each PSOM is trained separately, resulting in different weight values for different PSOMs. In contrast, for our cooperative EKMs, all EKMs are trained simultaneously to obtain the same input weight values (Section 3.5). Moreover, training of our EKMs is performed online rather than offline, as is the case for PSOM.

In the absence of precise quantitative error signals for training, reinforcement learning algorithms can be used if qualitative feedback signals are available. Nevertheless, they suffer from problems of generalization and continuity. Many reinforcement learning methods encode discrete sensory states and motor commands (Dietterich, 2000; Mahadevan and Connell, 1992; Rohanimanesh and Mahadevan, 2003), which cannot apply directly to the continuous sensorimotor domains of the real-world control tasks. A priori discretization of the continuous space may introduce hidden states and weak generalization, if done poorly. By combining with function approximators (e.g., MLP or feature map) that are capable of generalizing across continuous sensory input and motor control output spaces (Baird, 1995; Gross et al., 1998; Millán et al., 2002; Santamária et al., 1998; Smart and Kaelbling, 2000; Smith, 2002; Touzet, 1997), this limitation can be overcome. However, generalizing with function approximators does not guarantee that the algorithms will learn to produce continuous motor commands that vary smoothly and accurately in response to continuous changes in sensory state. In effect, some reinforcement learning algorithms (Millán et al., 2002; Santamária et al., 1998; Smith, 2002; Touzet, 1997) that are combined with function approximators map from continuous sensory input space to discrete motor control commands, which is exactly what the direct- 
mapping EKM does (Section 3.1). The drawbacks of such a continuity problem will be demonstrated in Section 4.1. To resolve this problem, some methods (Baird, 1995; Gross et al., 1998; Smart and Kaelbling, 2000) map to continuous motor control space but are burdened by very slow iterative search for the optimal action.

\section{Ensemble of Cooperative EKMs}

\subsection{Overview}

An EKM is a neural network that extends Kohonen's SOFM (Kohonen, 2000). Its self-organization of the input space is similar to Voronoi tessellation such that each tessellated region is encoded by the input weights of an EKM neuron. In addition to encoding a set of input weights that self-organize the sensory input space, the EKM neurons also produce outputs that vary with the incoming sensed inputs. The EKMs described in this paper adopt an egocentric representation of the sensory input vector: $\mathbf{u}(t)=\{\alpha, d\}^{T}$ where $\alpha$ and $d$ are the direction and the distance of a target location relative to the robot's current location and heading. At the goal state at time $T, \mathbf{u}(T)=(\alpha, 0)^{T}$ for any $\alpha$.

In many proposed EKMs (Cameron et al., 1998; Heikkonen and Koikkalainen, 1997; Rao and Fuentes, 1998; Ritter and Schulten, 1986; Smith, 2002; Touzet, 1997; Versino and Gambardella, 1995), each sensory input $\mathbf{u}$ is mapped directly to a motor control command $c$. In such a direct-mapping EKM (Fig. 1A), each neuron $i$ has a sensory weight vector $\mathbf{w}_{i}=\left(\alpha_{i}, d_{i}\right)^{T}$ that encodes a tessellated region in $\mathcal{U}$ centered at $\mathbf{w}_{i}$. It also has a weight vector $\mathbf{c}_{i}$ that encodes the motor control outputs produced by the neuron. With an incoming sensory input $\mathbf{u}$, the winning neuron $s$ is determined such that its sensory weight vector $\mathbf{w}_{s}$ is nearest to $\mathbf{u}$ (Eq. 2). This winning neuron $s$ outputs its motor control vector $\mathbf{c}_{s}$ to move the robot (Fig. 1A). Note that any incoming sensory input $\mathbf{u}$ that lies within the tessellated region encoded by $\mathrm{w}_{s}$ will produce the same motor control vector $\mathbf{c}_{s}$.

If sensorimotor control is a linear problem, then the motor control vector $\mathbf{c}$ would be related to the sensory input vector $\mathbf{u}$ by the linear equation:

$$
\mathbf{c}=\mathrm{Mu}
$$

where $\mathrm{M}$ is a matrix of motor control parameters. The control problem would be reduced to one of determining $M$ from the training samples. 


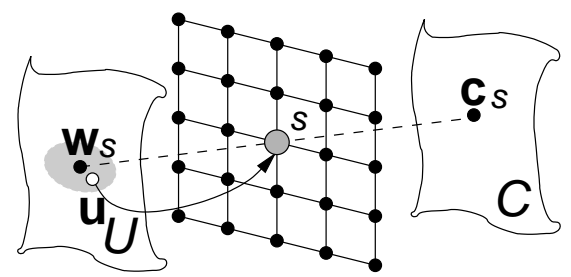

A

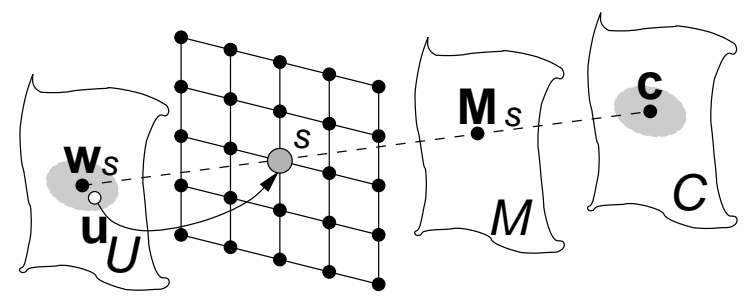

B

Figure 1: EKM architectures. (A) Neurons of a direct-mapping EKM map the sensory input space $\mathcal{U}$ directly to discretized points in the motor control space $\mathcal{C}$. (B) Neurons of an indirect-mapping EKM map the sensory input space $\mathcal{U}$ indirectly to the continuous motor control space $\mathcal{C}$ through the control parameter space $\mathcal{M}$. It resembles the EKM model of (Walter and Schulten, 1993), which stores both motor control vectors and matrices of control parameters as output weights. On the other hand, the indirect-mapping EKM only stores matrices of control parameters as output weights.

In practice, however, sensorimotor coordination is typically a nonlinear problem because a real motor takes a finite but non-zero amount of time to accelerate or decelerate in order to change speed. This problem is exacerbated in non-holonomic robots. A non-holonomic robot has restrictions in the way it can move due to kinematic or dynamic constraints such as limited turning abilities or momentum at high velocities (e.g., car) (Arkin, 1998). Hence, a nonholonomic robot is much harder to control and to achieve smooth motion than a holonomic robot (Russell and Norvig, 1995).

To solve the nonlinear problem, our indirect-mapping EKM (Low et al., $2002)$ is trained to partition the sensory input space $\mathcal{U}$ into locally linear regions. Each neuron $i$ in the EKM has a sensory weight vector $\mathbf{w}_{i}$ similar to that of a neuron in the direct-mapping EKM. However, unlike the direct-mapping approach, the output weights of neuron $i$ represent control parameters $\mathbf{M}_{i}$ in the parameter space $\mathcal{M}$ (Fig. 1B) instead of the motor control vector $\mathbf{c}_{i}$. The control parameter matrix $\mathbf{M}_{i}$ is mapped to the actual motor control vector $\mathbf{c}$ by the linear model of Eq. 1.

To elaborate, the direct-mapping approach maps all the sensory inputs $\mathbf{u}$ in a tessellated region in the sensory input space $\mathcal{U}$, represented by a neuron $s$, to the same discrete point $\mathbf{c}_{s}$ in the motor output space $\mathcal{C}$, i.e., $\mathbf{c}=\mathbf{c}_{s}$. Thus, only a small number of points in $\mathcal{C}$ are represented by the neurons' outputs, i.e., $\mathcal{C}$ is very sparsely sampled. In contrast, our indirect-mapping approach maps 


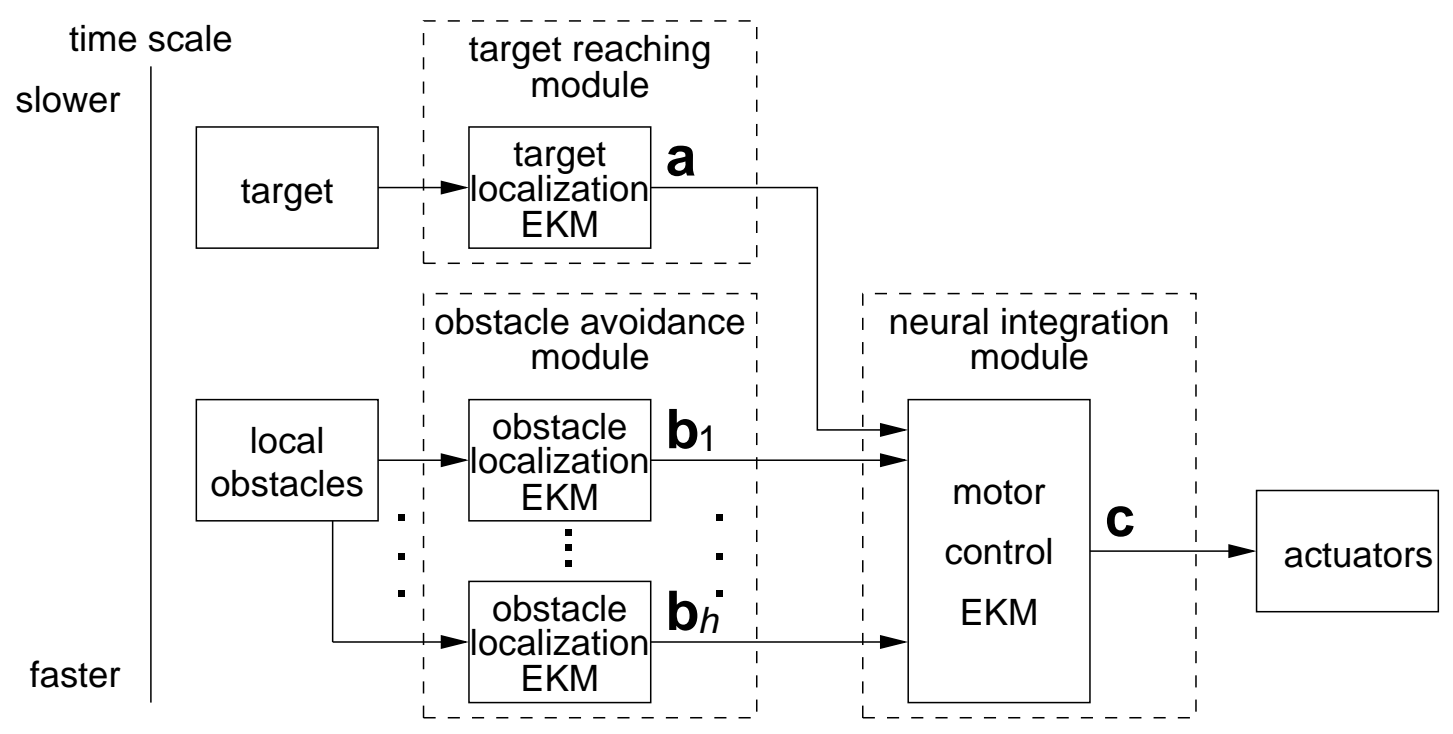

Figure 2: Framework of Cooperative EKMs.

each $\mathbf{u}$ in a local region in $\mathcal{U}$ to a different point $\mathbf{c}$ in $\mathcal{C}$ through Eq. 1 . Since this mapping is linear and continuous, the indirect-mapping approach maps a region in $\mathcal{U}$ to a region in $\mathcal{C}$ (Fig. 1B). This method permits finer, smoother, and more efficient sensorimotor control of the robot's target reaching motion compared to the direct-mapping approach (Section 4.1).

Cooperative EKMs (Low et al., 2003) are implemented by connecting an ensemble of EKMs into three modules: target reaching, obstacle avoidance, and neural integration (Fig. 2). The target localization EKM in the target reaching module is activated by the presence of a target within the robot's target sensing range. The EKM receives a sensed target location and outputs corresponding excitatory signals to the motor control EKM in the neural integration module at and around the locations of the sensed target.

The obstacle localization EKMs in the obstacle avoidance module are activated by the presence of obstacles within the robot's obstacle sensing range. Each EKM receives a sensed obstacle location and outputs corresponding inhibitory signals to the motor control EKM in the neural integration module at and around the locations of the sensed obstacles.

The motor control EKM in the neural integration module serves as the sensorimotor interface, which integrates the activity signals from the EKMs for cooperation and competition to produce an appropriate motor signal to the actuators. This motor signal allows a robot to approach a target and negotiate 
obstacles.

The cooperative EKMs framework allows the modules to operate asynchronously at different rates, which is the key to preserving reactive capabilities. For example, the target reaching module operates at about $256 \mathrm{~ms}$ between servo ticks while the obstacle avoidance module can typically operate faster at intervals of $128 \mathrm{~ms}$. The neural integration module is activated as and when neural activities are received.

\subsection{Target Reaching}

The target reaching module uses the target localization EKM to self-organize the sensory input space $\mathcal{U}$. Each neuron $i$ in the EKM has a sensory weight vector $\mathbf{w}_{i}=\left(\alpha_{i}, d_{i}\right)^{T}$ that encodes a region in $\mathcal{U}$ centered at $\mathbf{w}_{i}$. Based on each incoming sensory input $\mathbf{u}$ of the target location, the target localization EKM outputs excitatory signals to the motor control EKM in the neural integration module (Section 3.4). The target localization EKM is activated as follows:

\section{Target Localization}

Given a sensory input $\mathbf{u}$ of a target location,

1. Determine the winning neuron $s$ in the target localization EKM. The winning neuron $s$ is the one whose sensory weight vector $\mathbf{w}_{s}=\left(\alpha_{s}, d_{s}\right)^{T}$ is nearest to the input $\mathbf{u}=(\alpha, d)^{T}$ :

$$
D\left(\mathbf{u}, \mathbf{w}_{s}\right)=\min _{i \in \mathcal{A}(\alpha)} D\left(\mathbf{u}, \mathbf{w}_{i}\right) .
$$

The difference $D\left(\mathbf{u}, \mathbf{w}_{i}\right)$ is a weighted difference between $\mathbf{u}$ and $\mathbf{w}_{i}$ :

$$
D\left(\mathbf{u}, \mathbf{w}_{i}\right)=\beta_{\alpha}\left(\alpha-\alpha_{i}\right)^{2}+\beta_{d}\left(d-d_{i}\right)^{2}
$$

where $\beta_{\alpha}$ and $\beta_{d}$ are constant parameters. The minimum in Eq. 2 is taken over the set $\mathcal{A}(\alpha)$ of neurons encoding very similar angles as $\alpha$ :

$$
\left|\alpha-\alpha_{i}\right| \leq\left|\alpha-\alpha_{j}\right|, \quad \text { for each pair } i \in \mathcal{A}(\alpha), j \notin \mathcal{A}(\alpha) \text {. }
$$

In other words, direction has priority over distance in the competition between EKM neurons. This method allows the robot to quickly orientate itself to face the target while moving towards it. An EKM contains a limited set of neurons, each of which has a sensory weight vector $\mathbf{w}_{i}$ that encodes a point in the sensory input space $\mathcal{U}$. The region in $\mathcal{U}$ that encloses all the sensory weight vectors of these neurons is called the local workspace $\mathcal{U}^{\prime}$. Even if the target falls outside $\mathcal{U}^{\prime}$, the nearest neuron can still be activated (Fig. 3A). 


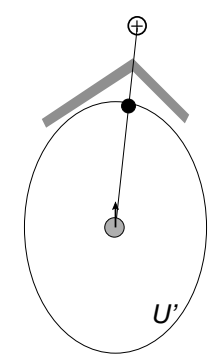

A

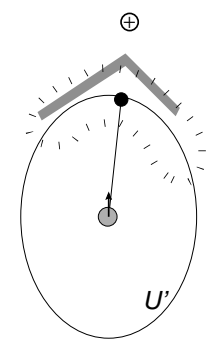

B

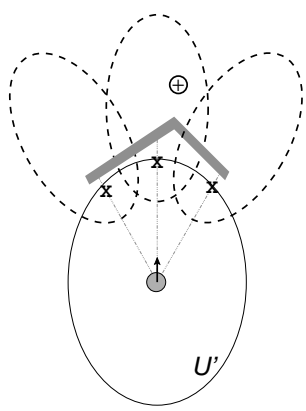

C

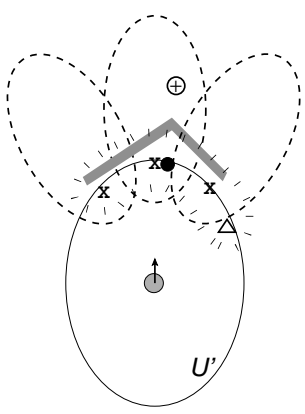

D

Figure 3: Conceptual description of cooperative EKMs. (A) In response to the target $\oplus$, the nearest neuron (black dot) in the target localization EKM (ellipse) of the robot (gray circle) is activated. (B) The activated neuron produces a target field (dotted region) in the motor control EKM. (C) Three of the robot's sensors detect obstacles and activate three neurons (crosses) in the obstacle localization EKMs, which produce the obstacle fields (dashed ellipses). (D) Subtraction of the obstacle fields from the target field results in the neuron at $\triangle$ to become the winner in the motor control EKM, which moves the robot away from the obstacle.

2. Compute output activity $a_{i}$ of neuron $i$ in the target localization EKM.

$$
a_{i}=G_{a}\left(\mathbf{w}_{s}, \mathbf{w}_{i}\right) .
$$

The function $G_{a}$ is an elongated Gaussian:

$$
G_{a}\left(\mathbf{w}_{s}, \mathbf{w}_{i}\right)=\exp \left(-\frac{\left(\alpha_{s}-\alpha_{i}\right)^{2}}{2 \sigma_{a \alpha}^{2}}-\frac{\left(d_{s}-d_{i}\right)^{2}}{2 \sigma_{a d}^{2}}\right) .
$$

Parameter $\sigma_{a d}$ is much smaller than $\sigma_{a \alpha}$, making the Gaussian distancesensitive and angle-insensitive. These parameter values elongate the Gaussian along the direction perpendicular to the target direction $\alpha_{s}$ (Fig. 3B). This elongated Gaussian is the target field, which plays an important role in overcoming concave obstacles. The effects of these parameters on the robot's target reaching capabilities will be examined in Section 4.2.

The output activities of the neurons in the target localization EKM are aggregated in the motor control EKM to produce a motion that moves the robot towards the target. This will be explained in Section 3.4. In the next section, we will present the obstacle localization EKMs, which are activated in a similar manner as the target localization EKM. 


\subsection{Obstacle Avoidance}

The obstacle avoidance module uses obstacle localization EKMs. The robot has $h$ directed distance sensors around its body for detecting obstacles. Hence, each activated sensor encodes a fixed direction $\alpha_{j}$ and a variable distance $d_{j}$ of the obstacle relative to the robot's heading and location. Each sensor's input $\mathbf{u}_{j}=\left(\alpha_{j}, d_{j}\right)^{T}$ induces an obstacle localization EKM. Note that each distance sensor (e.g., laser) can only reflect the nearest obstacle in its sensing direction. Hence, the number of obstacle localization EKMs that are activated does not depend on the number of obstacles but rather, on the number of distance sensors. The obstacle localization EKMs have the same number of neurons and input weight values as the target localization EKMs, i.e., each neuron $i$ in the obstacle localization EKM has the same input weight vector $\mathbf{w}_{i}$ as the neuron $i$ in the target localization EKM. The EKMs output inhibitory signals to the motor control EKM in the neural integration module (Section 3.4). The obstacle localization EKMs are activated as follows:

\section{Obstacle Localization}

For each sensory input $\mathbf{u}_{j}, j=1, \ldots, h$ (i.e., $h$ distance sensors),

1. Determine the winning neuron $s$ in the $j$ th obstacle localization EKM. The obstacle localization EKM is activated in the same manner as Step 1 of Target Localization (Section 3.2).

2. Compute output activity $b_{i}$ of neuron $i$ in the $j$ th obstacle localization EKM:

$$
b_{i}=G_{b}\left(\mathbf{w}_{s}, \mathbf{w}_{i}\right)
$$

where

$$
\begin{aligned}
G_{b}\left(\mathbf{w}_{s}, \mathbf{w}_{i}\right) & =\exp \left(-\frac{\left(\alpha_{s}-\alpha_{i}\right)^{2}}{2 \sigma_{b \alpha}^{2}}-\frac{\left(d_{s}-d_{i}\right)^{2}}{2 \sigma_{b d}^{2}\left(d_{s}, d_{i}\right)}\right) \\
\sigma_{b d}\left(d_{s}, d_{i}\right) & = \begin{cases}2.475 & \text { if } d_{i} \geq d_{s} \\
0.02475 & \text { otherwise. }\end{cases}
\end{aligned}
$$

The function $G_{b}$ is a Gaussian stretched along the obstacle direction $\alpha_{s}$ so that motor control EKM neurons beyond the obstacle locations are also inhibited to indicate inaccessibility (Fig. 3C). If no obstacle is detected, $G_{b}=0$. In the presence of an obstacle, the neurons in the obstacle localization EKMs at and near the obstacle locations will be activated to produce obstacle fields. The neurons nearest to the obstacle locations have the strongest activities. The effects of the parameters $\sigma_{b d}$ and $\sigma_{b \alpha}$ on the robot's obstacle avoidance capabilities will be investigated in Section 4.2. 


\subsection{Neural Integration and Motor Control}

The neural integration module uses a motor control EKM to integrate the activities from the neurons in the target and obstacle localization EKMs. The motor control EKM has the same number of neurons and input weight values as the target and robot localization EKMs. The neural integration is performed as follows:

\section{Neural Integration}

1. Compute activity $e_{i}$ of neuron $i$ in the motor control EKM.

$$
e_{i}=a_{i}-\sum_{j=1}^{h} b_{j i}
$$

where $a_{i}$ is the excitatory input from neuron $i$ of the target localization EKM (Section 3.2) and $b_{j i}$ is the inhibitory input from neuron $i$ of the $j$-th obstacle localization EKM (Section 3.3).

2. Determine the winning neuron $k$ in the motor control EKM. Neuron $k$ is the one with the largest activity:

$$
e_{k}=\max _{i} e_{i}
$$

The motor control EKM also has a set of output weights, which encode the outputs produced by the neuron. However, unlike existing direct-mapping methods (Cameron et al., 1998; Heikkonen and Koikkalainen, 1997; Rao and Fuentes, 1998; Ritter and Schulten, 1986; Smith, 2002; Touzet, 1997; Versino and Gambardella, 1995), the output weights of neuron $i$ of the motor control EKM represent control parameters $\mathbf{M}_{i}$ in the parameter space $\mathcal{M}$ instead of the actual motor control vector (Fig. 1). The control parameter matrix $\mathbf{M}_{i}$ is mapped to the actual motor control vector $\mathrm{c}$ by a linear model (Eq. 11). With indirect-mapping EKM, motor control is performed as follows:

\section{Motor Control}

Compute motor control vector c:

$$
\mathbf{c}= \begin{cases}\mathbf{M}_{k} \mathbf{u} & \text { if }\left|\mathbf{M}_{k} \mathbf{u}\right| \leq \mathbf{c}^{*} \text { and } k=s \\ \mathbf{M}_{k} \mathbf{w}_{k} & \text { otherwise }\end{cases}
$$

where $s$ is the winning neuron in the target localization EKM, and $\mathbf{M}_{k}$ and $\mathbf{w}_{k}$ are, respectively, the control parameter matrix and sensory weight vector of the winning neuron $k$ in the motor control EKM (Step 2 of Neural Integration). 
The constant vector $\mathbf{c}^{*}$ denotes the upper limit of physically realizable motor control signal. For instance, for the Khepera robots, c consists of the motor speeds $v_{l}$ and $v_{r}$ of the robot's left and right wheels. In this case, we define $\mathbf{c} \leq \mathbf{c}^{*}$ if $v_{l} \leq v_{l}^{*}$ and $v_{r} \leq v_{r}^{*}$. Note that if $\mathbf{c}$ is beyond $\mathbf{c}^{*}$, simply saturating the wheel speeds does not work. For example, if the target is far away and not aligned with the robot's heading, then saturating both wheel speeds only moves the robot forward. Without correcting the robot's heading, the robot will not be able to reach the target. Hence, the winning neuron's input weights $\mathbf{w}_{k}$ are used to generate the physically realizable motor control output. This motor control would be the best substitution for the sensory input $\mathbf{u}$ because $\mathbf{w}_{k}$ is closest to $\mathbf{u}$ compared to other weights $\mathbf{w}_{i}, i \neq k$.

In activating the motor control EKM (Fig. 3D), the obstacle fields are subtracted from the target field (Eq. 9). If the target lies within the obstacle fields, the activation of the motor control EKM neurons close to the target location will be suppressed. Consequently, another neuron at a location that is not inhibited by the obstacle fields becomes most highly activated (Fig. 3D). This neuron produces a control parameter that moves the robot away from the obstacle. While the robot moves around the obstacle, the target and obstacle localization EKMs are continuously updated with the current locations and directions of the target and obstacles. Their interactions with the motor control EKM produce fine, smooth, and accurate motion control of the robot to negotiate the obstacle and move towards the target until it reaches the goal state $\mathbf{u}(T)$ at time step $T$.

Figure 4 shows the output activities of the neurons in different EKMs produced in response to the environment setup depicted in Fig. 3. In Fig. 4A, the output activities of the neurons in the target localization EKM form the target field (Fig. 3B). Since the neuron at $d=0.16 \mathrm{~m}$ and $\alpha=0.1$ radian (darkest dot in Fig. 4A) is closest to the target location, it is most strongly activated and thus produces the highest output activity. This neuron corresponds to the black dot in Fig. 3B. Its neighboring neurons also produce relatively strong output activities to form the target field used in overcoming the concave obstacle. The obstacle localization EKMs shown in Figs. 4B, 4C, and 4D are activated by distance sensors positioned at $-\pi / 6,0$, and $\pi / 6$ radian respectively. For each EKM induced by a sensor, the neuron that is closest to its sensed obstacle becomes most strongly activated. These activated neurons are at $d=0.132 \mathrm{~m}$ and $\alpha=-0.4$ radian (darkest dot in Fig. 4B), $d=0.165 \mathrm{~m}$ and $\alpha=0$ radian (darkest dot in Fig. 4C), and $d=0.139 \mathrm{~m}$ and $\alpha=0.4$ radian (darkest dot in Fig. 4D). They correspond to the three crosses in Fig. 3C. Figure 4E shows the combined output activities of the neurons in the obstacle localization EKMs, which form the obstacle fields (Fig. 3C). Since the target lies within the obstacle fields, the 


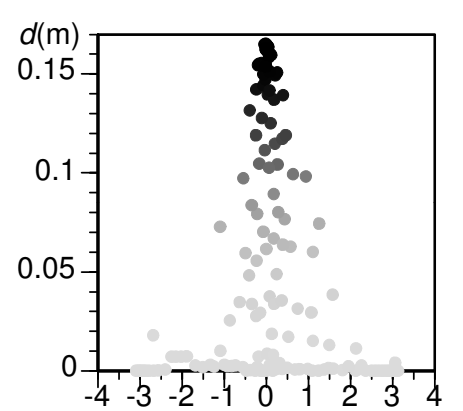

A

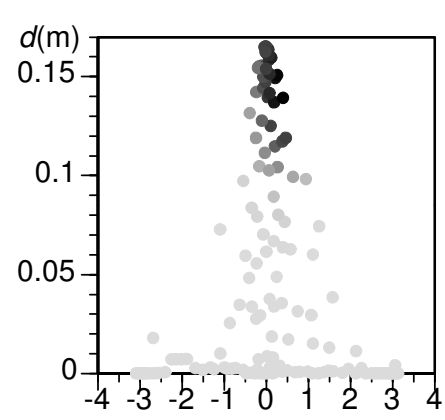

D

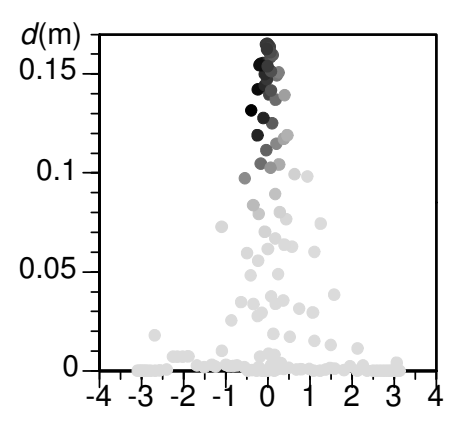

B

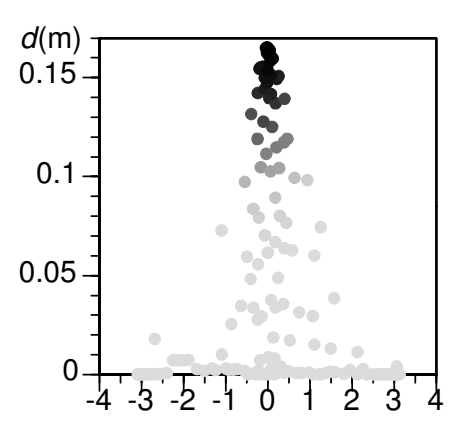

E

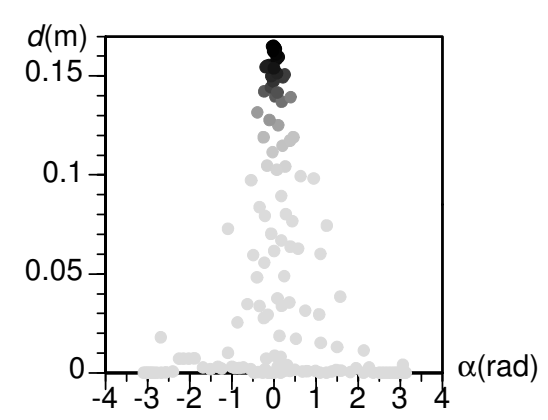

C

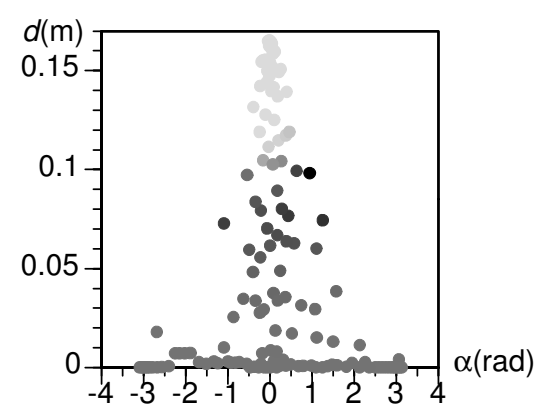

$\mathbf{F}$

Figure 4: Output activities of neurons in (A) target localization EKM, (B) obstacle localization EKM activated by distance sensor at $-\pi / 6$ radian, $(\mathrm{C})$ obstacle localization EKM activated by distance sensor at 0 radian, (D) obstacle localization EKM activated by distance sensor at $\pi / 6$ radian, (E) obstacle localization EKMs combined, and (F) localization EKMs combined during neural integration. Each dot denotes the sensory weights $\mathbf{w}_{i}=\left(\alpha_{i}, d_{i}\right)^{T}$ of a neuron. A darker dot implies that the neuron has a stronger output activity. A lighter dot implies the opposite.

strong excitatory activities from the target localization EKM neurons that are close to the target location will be suppressed. As a result, another neuron at $d=0.098 \mathrm{~m}$ and $\alpha=0.9$ radian (darkest dot in Fig. $4 \mathrm{~F}$ ) that is not inhibited by the obstacle fields becomes most strongly activated in the motor control EKM. This neuron corresponds to $\triangle$ in Fig. 3D. It produces a control parameter that enables the robot to negotiate the concave obstacle.

Recall that the various modules run asynchronously at different rates (Section 3.1). In particular, the obstacle avoidance module runs at a faster rate than 
the target reaching module. During neural integration, the localization EKMs remain activated until they are updated asynchronously at the next sensing cycle. So, the motor control EKM can receive continuous inputs from the localization EKMs and is always able to produce a motor signal as and when new inputs are sensed.

\subsection{Self-Organization of EKMs}

In contrast to most existing offline learning methods (Bruske and Sommer, 1995; Gorinevsky and Connolly, 1994; Karayiannis and Mi, 1997; Moody and Darken, 1989), online learning is adopted for the EKMs. Initially, the EKMs have not been trained and the motor control vectors $\mathbf{c}$ generated are inaccurate. Nevertheless, the EKMs self-organize, using these control vectors $\mathbf{c}$ and the corresponding robot displacements $\mathbf{v}$ produced by $\mathbf{c}$, to map $\mathbf{v}$ to $\mathbf{c}$ indirectly. Note that $\mathbf{v}$ is used as the training input rather than sensory input $\mathbf{u}$. Since the untrained EKMs produce inaccurate motor control vectors $\mathbf{c}$ in response to $\mathbf{u}$ (i.e., $\mathbf{c}$ does not move the robot to the target location specified by $\mathbf{u})$, the robot will learn the wrong sensorimotor mapping if $\mathbf{u}$ is used as the corresponding training input. On the other hand, $\mathrm{v}$ is the actual displacement that corresponds to $\mathbf{c}$. Using $\mathbf{v}$ as the training input will enable the robot to learn the correct mapping as it moves around. Hence, its sensorimotor control becomes more accurate. At this stage, the online learning just fine-tunes the indirect mapping. The self-organized learning algorithm (in an obstacle-free environment) is as follows:

\section{Self-Organized Learning}

Repeat

1. Get sensory input $\mathbf{u}$.

2. Execute target reaching procedure and move robot.

3. Get new sensory input $\mathbf{u}^{\prime}$ and compute actual displacement $\mathbf{v}$ as a difference between $\mathbf{u}^{\prime}$ and $\mathbf{u}$.

4. Use $\mathbf{v}$ as the training input to determine the winning neuron $k$ (same as Step 1 of Target Localization except that $\mathbf{u}$ is replaced by $\mathbf{v}$ ).

5. Adjust the weights $\mathbf{w}_{i}$ of neurons $i$ in the neighborhood $\mathcal{N}_{k}$ of the winning neuron $k$ towards v:

$$
\Delta \mathbf{w}_{i}=\eta G(k, i)\left(\mathbf{v}-\mathbf{w}_{i}\right)
$$


where $G(k, i)$ is a Gaussian function of the distance between the positions of neurons $k$ and $i$ in the EKM, and $\eta$ is a constant learning rate. This step is similar to the self-organization of Kohonen's self-organizing map.

6. Update the weights $\mathbf{M}_{i}$ of neurons $i$ in the neighborhood $\mathcal{N}_{k}$ to minimize the error $e$ :

$$
e=\frac{1}{2} G(k, i)\left\|\mathbf{c}-\mathbf{M}_{i} \mathbf{v}\right\|^{2} .
$$

That is, apply a recursive stochastic approximation algorithm, which can be cast into this general form:

$$
\Delta \mathbf{M}_{i}=-\eta \frac{\partial e}{\partial \mathbf{M}_{i}} \mathbf{H}_{i}
$$

where $\mathbf{H}_{i}$ is a weighting matrix. If $\mathbf{H}_{i}=\mathbf{I}$, a first-order learning method, gradient descent, is obtained from Eq. 14:

$$
\Delta \mathbf{M}_{i}=-\eta \frac{\partial e}{\partial \mathbf{M}_{i}} \mathbf{I}=\eta G(k, i)\left(\mathbf{c}-\mathbf{M}_{i} \mathbf{v}\right) \mathbf{v}^{T} .
$$

In the case of the quadratic error function $e$ (Eq. 13), learning can be accelerated by a second-order learning method (Battiti, 1992). This can be achieved by setting $\mathbf{H}_{i}$ to $\mathbf{R}_{i}^{-1}$ where $\mathbf{R}_{i}$ is a Gauss-Newton approximation of the Hessian $\partial^{2} e / \partial \mathbf{M}_{i}^{2}$. A second-order learning method, recursive least squares (Glentis et al., 1999), is thus derived from Eq. 14 with $\eta=1$ (optimum step size):

$$
\begin{aligned}
\Delta \mathbf{R}_{i}^{-1} & =\frac{1}{\lambda}\left((1-\lambda) \mathbf{R}_{i}^{-1}-\frac{\mathbf{R}_{i}^{-1} \mathbf{v} \mathbf{v}^{T} \mathbf{R}_{i}^{-1}}{\frac{\lambda}{G(k, i)}+\mathbf{v}^{T} \mathbf{R}_{i}^{-1} \mathbf{v}}\right) \\
\Delta \mathbf{M}_{i} & =-\frac{\partial e}{\partial \mathbf{M}_{i}} \mathbf{R}_{i}^{-1}=G(k, i)\left(\mathbf{c}-\mathbf{M}_{i} \mathbf{v}\right) \mathbf{v}^{T} \mathbf{R}_{i}^{-1}
\end{aligned}
$$

where $\lambda$ is a constant forgetting rate and $\mathbf{R}_{i}^{-1}$ is initialized to $\mathbf{I}$. Note that the recursive online update of $\mathbf{R}_{i}^{-1}$ (Eq. 16) is obtained using matrix inversion lemma to avoid the costly matrix inversion operation (Haykin, 2002). Each update of $\mathbf{M}_{i}$ requires $O\left(n^{2}\right)$ computations and $O\left(n^{2}\right)$ additional memory to store $\mathbf{R}_{i}^{-1}$ where $n$ is the number of dimensions in $\mathbf{v}$. In contrast, gradient descent requires $O(n)$ computations and no additional memory. The performance of these two learning methods is compared in Section 4.1. 
The target and obstacle localization EKMs self-organize in the same manner as the motor control EKM except that Step 6 is omitted. At each training cycle, the weights of the winning neuron $k$ and its neighboring neurons $i$ are modified. The amount of modification is proportional to the distance $G(k, i)$ between the neurons in the EKM. The input weights $\mathbf{w}_{i}$ are updated towards the actual displacement $\mathbf{v}$ and the control parameters $\mathbf{M}_{i}$ are updated so that they map the displacement $\mathbf{v}$ to the corresponding motor control c. After self-organization has converged, the neurons will stabilize in a state such that $\mathbf{v}=\mathbf{w}_{i}$ and $\mathbf{c}=\mathbf{M}_{i} \mathbf{v}=\mathbf{M}_{i} \mathbf{w}_{i}$. For any winning neuron $k$, given that $\mathbf{u}=\mathbf{w}_{k}$, the neuron will produce a motor control output $\mathbf{c}=\mathbf{M}_{k} \mathbf{w}_{k}$, which yields a desired displacement of $\mathbf{v}=\mathbf{w}_{k}$. If $\mathbf{u} \neq \mathbf{w}_{k}$ but close to $\mathbf{w}_{k}$, the motor output $\mathbf{c}=\mathbf{M}_{k} \mathbf{u}$ produced by neuron $k$ will still yield the correct displacement if linearity holds within the input region that activates neuron $k$. Thus, given enough neurons to produce an approximate linearization of the sensory input space $\mathcal{U}$, indirect-mapping EKM can produce finer and smoother motion control than direct-mapping EKM as shown in Section 4.1.

\section{Experiments and Discussion}

\subsection{Online Learning of Target Reaching Motion}

This section presents a quantitative evaluation of the indirect-mapping EKM in online sensorimotor learning of the robot's target reaching motion. For the purpose of evaluating performance, the following network architectures were compared:

1. B15: BFN with $15 \times 15$ neurons

2. D15: direct-mapping $\mathrm{EKM}$ with $15 \times 15$ neurons

3. G9: indirect-mapping EKM with $9 \times 9$ neurons trained by gradient descent

4. G12: indirect-mapping EKM with $12 \times 12$ neurons trained by gradient descent

5. G15: indirect-mapping $\mathrm{EKM}$ with $15 \times 15$ neurons trained by gradient descent

6. R15: indirect-mapping EKM with $15 \times 15$ neurons trained by recursive least squares 
Our implementation of BFN was similar to those proposed by Bruske and Sommer (1995), Hartman and Keeler (1991), Karayiannis and Mi (1997), and Moody and Darken (1989), except that it was trained online rather than offline. The basis function centers were trained in a similar manner as the input weights of indirect-mapping EKM (Eq. 12). Each basis function width was updated to approach the Euclidean distance between itself and its nearest neighbor (Hartman and Keeler, 1991; Moody and Darken, 1989; Platt, 1991). The output weights were trained via gradient descent.

We also attempted to train the basis function centers with gradient descent (Ghosh and Nag, 2001; Karayiannis, 1999; Platt, 1991; Poggio and Girosi, 1990; Wettschereck and Dietterich, 1992) but learning was unsuccessful despite extensive tuning of parameters. Although the robot learned to move towards the target locations successfully, it was not able to come to a stop at these locations, even after prolonged training. One possible explanation, as detailed by Moody and Darken (1989), is that gradient descent training of the basis function centers may lead to unpredictable target reaching motions because the centers are sometimes squeezed out of the region of input space that contain data. Furthermore, learning converges slowly due to non-linear optimization. In contrast, the self-organization of the input space in our implemented BFN is datacentric. More neurons are committed to input regions with dense sampling of data during online learning, which improves the resolution in these regions (Section 1). Faster convergence in learning has also been reported in this case (Moody and Darken, 1989).

The tests were performed using Webots (http://www.cyberbotics.com), a 3D, kinematic, sensor-based simulator for Khepera mobile robots, which incorporates $10 \%$ white noise in its sensors and actuators. The simulator computes the trajectories and sensory inputs of a robot situated in an environment corresponding to a given physical setup. The resulting simulation allows the controller to be transferred to a real robot without changes (Michel, 2004). The simulated behaviors are very close to those of a real robot, as demonstrated in these works (Hayes et al., 2002; Ijspeert et al., 2001; Martinoli et al., 1999).

In the experiments, the neural networks were trained in a $5 \mathrm{~m}$ by $5 \mathrm{~m}$ obstacle-free environment. Each training/testing trial took 100,000 time steps and each time step for target reaching motion lasted $1.024 \mathrm{sec}$. During training, the input weights were initialized to correspond to regularly spaced locations in the sensory input space $\mathcal{U}$. The robot began its network training at the center of the environment and a randomly selected sequence of targets was presented. The robot's task was to move to the targets, one at a time, and weight modification was performed at each time step after the robot had made a move. At 
each time interval of 10,000 steps during training, a fixed testing procedure was conducted. In each test, the robot began at the center of the environment and was presented with 50 random target locations in sequence. The robot's task was to move to each of the target locations. No training was performed during this testing phase. The above training/testing trial was repeated 5 times and testing performance was averaged over the 5 trials.

Three testing performance indices are measured in the above training/testing trials. The first index is the mean positioning error $E$, which measures the average distance $\varepsilon_{i}$ between the center of the robot and the $i$ th target location after it has come to a stop (i.e., motor control $\mathrm{c}=\mathbf{0}$ ):

$$
E=\frac{1}{R N} \sum_{i} \varepsilon_{i}
$$

where $R$ is the number of trials and $N$ is the number of testing target locations. The second index normalized time-to-target $T$ measures how long it takes the robot to reach the target locations:

$$
T=\frac{1}{R N} \sum_{i} \tilde{t}_{i}, \quad \tilde{t}_{i}=\frac{t_{i}}{l_{i}}
$$

where $t_{i}$ is the time it takes the robot to reach the $i$ th target, $l_{i}$ is the straight line distance between targets $i-1$ and $i$, and $\tilde{t}_{i}$ is the normalized time taken to reach target $i$. That is, normalized time-to-target measures the average amount of time the robot takes to travel a distance of $1 \mathrm{~m}$ towards a target. The third index mean deviation from straight-line trajectory $D$ measures how straight or wavy is the robot's trajectory:

$$
D=\frac{1}{R N} \sum_{i} \tilde{\delta}_{i}, \quad \tilde{\delta}_{i}=\frac{\left|d_{i}-l_{i}\right|}{l_{i}}
$$

where $d_{i}$ is the distance traveled to reach the target location $i$, and $\tilde{\delta}_{i}$ is the deviation from straight-line trajectory for target $i$.

Figures 5A and 5B show respectively how the mean positioning error and normalized time-to-target decreased during the self-organized learning of various network architectures. In Fig. 5A, both B15 and D15 stabilized as early as 10,000 time steps but achieved much poorer $E$ performance compared to the other networks. On the other hand, G9, G12, G15, and R15 stabilized more gradually at about 70,000,60,000,50,000, and 15,000 time steps respectively but they could all achieve lower $E$. Notice that the larger indirect-mapping EKMs stabilized faster. To explain this counter-intuitive result, note that the standard 


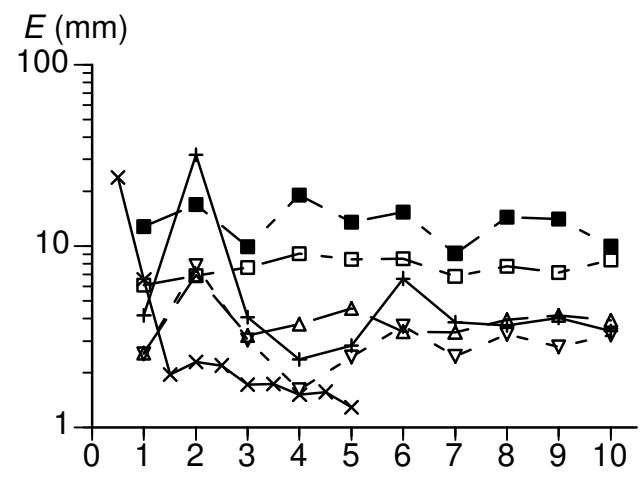

A

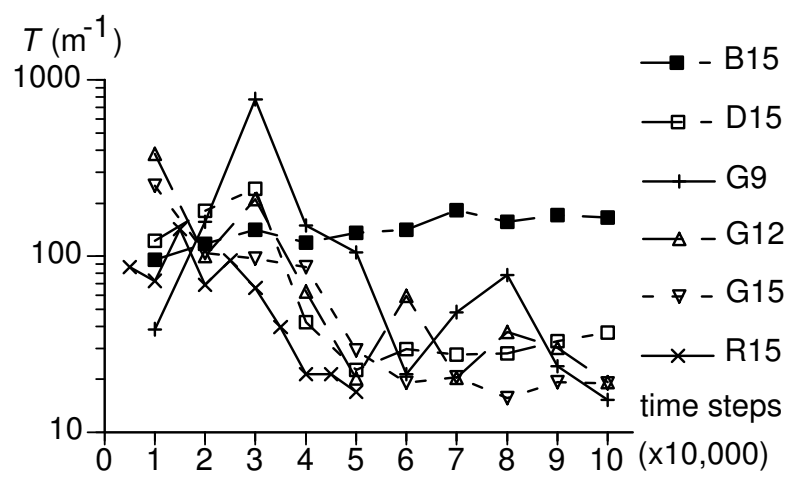

B

Figure 5: Performance comparison between various network architectures in (A) mean positioning error, and (B) normalized time-to-target.

deviations of the Gaussian functions in Equations 12 and 15 (Section 3.5) were the same for all EKMs. That is, the proportion of neurons requiring weight updates at each time step was greater in smaller EKMs than in larger EKMs. As such, the neurons in smaller EKMs updated their weights more frequently, thus stabilizing more slowly.

While the $E$ performance shows the quality of the robot positioning at the target location, the $T$ and $D$ performance demonstrate the quality of the robot's trajectory. We will only illustrate $T$ in Fig. 5B; the convergence of $D$ is similar. The self-organization of D15 stabilized at about 50,000 time steps. On the other hand, G9, G12, and G15 stabilized at about 90,000, 70,000, and 50,000 time steps respectively, which supported the above observation that larger EKMs stabilized more quickly. R15 stabilized at 40,000 time steps, which was faster than that trained by gradient descent. Therefore, its training/testing process was stopped at 50,000 time steps, which was sufficient for its self-organized learning to stabilize. Although B15 stabilized as early as 10,000 time steps, its poorer performance, relative to the other networks, became obvious with increasing training time.

Table 1 shows the test results after training. All indirect-mapping EKMs achieved lower mean positioning errors, normalized time-to-target and mean deviation from straight line trajectory than D15 and B15. R15 achieved much lower $E$ and $D$ than G9, G12, and G15. Among the indirect-mapping EKMs trained by gradient descent, G12 enabled the robot to travel the straightest path to stop at the target location. Reducing the number of neurons to $9 \times 9$ caused 
Table 1: Performance comparison between networks after training.

\begin{tabular}{ccrrc}
\hline Network & Total & \multicolumn{3}{c}{ Performance indices } \\
\cline { 3 - 5 } & parameters & \multicolumn{1}{c}{$E(\mathrm{~mm})$} & \multicolumn{1}{c}{$T\left(\mathrm{~m}^{-1}\right)$} & $D$ \\
\hline B15 & 1350 & $10.02 \pm 3.81$ & $166.40 \pm 28.49$ & $0.11 \pm 0.06$ \\
D15 & 900 & $8.37 \pm 2.25$ & $36.78 \pm 11.11$ & $0.18 \pm 0.15$ \\
G9 & 486 & $3.40 \pm 1.83$ & $15.31 \pm 4.64$ & $0.10 \pm 0.04$ \\
G12 & 864 & $3.89 \pm 1.61$ & $19.31 \pm 8.47$ & $0.06 \pm 0.02$ \\
G15 & 1350 & $3.23 \pm 0.66$ & $18.96 \pm 4.37$ & $0.07 \pm 0.02$ \\
R15 & 1350 & $1.29 \pm 0.14$ & $17.00 \pm 2.48$ & $0.04 \pm 0.01$ \\
\hline
\end{tabular}

the path to be more convoluted. Increasing the number of neurons to $15 \times 15$ increased, instead of decreased, $D$ slightly. This phenomenon could be explained by how the neurons self-organized in the sensory input space $\mathcal{U}$, which is elaborated in the next paragraph.

Neurons in G15 were self-organized into four clusters: $d=0 \mathrm{~m}$ and $\alpha=$ $-3,0,+3$ radian (Fig. 6C). On the other hand, neurons in G9 and G12 were selforganized into two clusters only: $d=0 \mathrm{~m}$ and $\alpha=0$ radian (Figs. 6A, 6B). With more neurons, G15 gained the flexibility of backward motion $(\alpha=-3,+3$ radian). However, these two regions of input space were less well sampled by the neurons than the region at $\alpha=0$ radian. As such, if a distant target appeared behind the robot with G15, its backward motion would produce a more wavy path. The robot with G12 would instead turn around to face the target via the cluster at $d=0 \mathrm{~m}$ before moving forward in a much straighter path. As for G9 (Fig. 6A), since its neurons sampled the input space at $\alpha=0$ radian more sparsely than those in $\mathrm{G} 15$ at $\alpha=0,+3,-3$ radian (i.e., both forward and backward motion), it would inevitably produce a more convoluted path than G15 regardless of whether the target is in front or behind.

Table 1 also shows that smaller mean deviation did not necessarily imply shorter normalized time-to-target. During learning, direction had priority over distance in the competition between EKM neurons (Eq. 4). So, a larger EKM had more neurons allocated for adjusting orientation without moving long distances (i.e., $d=0 \mathrm{~m}$ cluster in Fig. 6). Consequently, the robot might move short motion steps to adjust its orientation first before moving straight to the target. Therefore, its trajectory deviated less from the straight line path.

The advantages of indirect-mapping EKMs over D15 and B15 can also be assessed from the self-organization results (Fig. 6). The neurons in the indirect- 


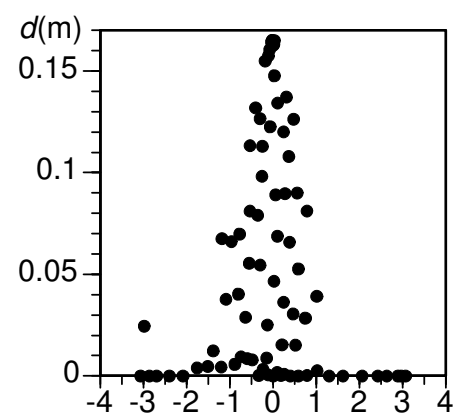

A

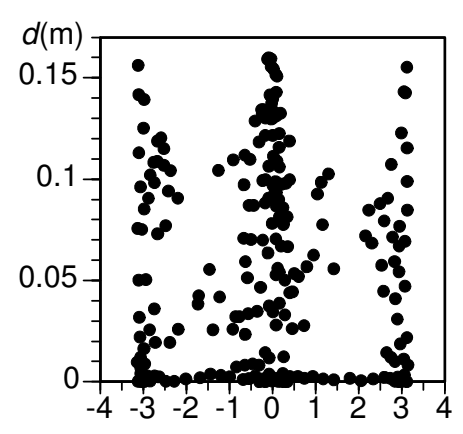

D

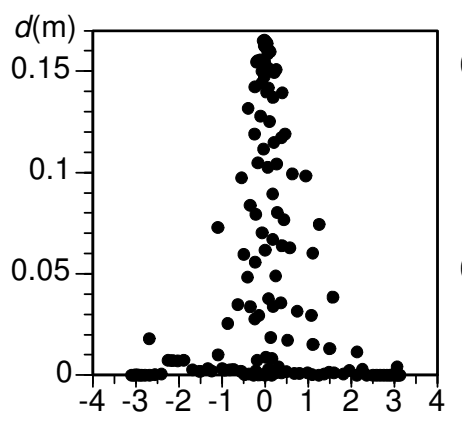

B

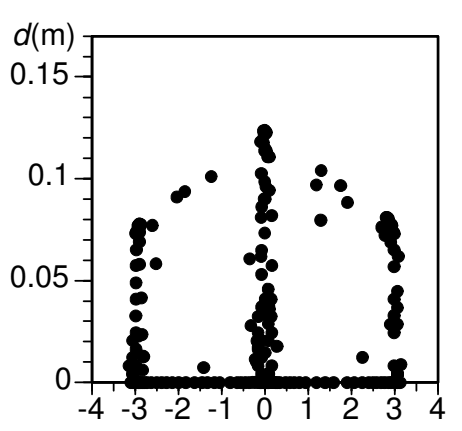

E

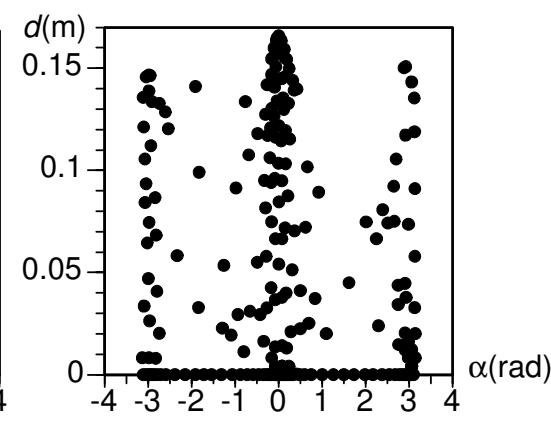

C

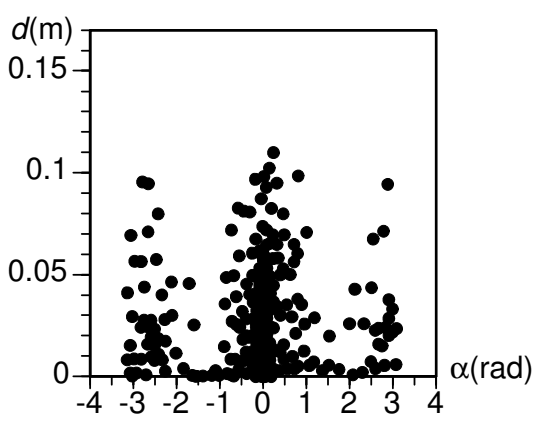

$\mathbf{F}$

Figure 6: Self-organization results of (A) G9, (B) G12, (C) G15, (D) R15, (E) D15, and (F) B15 taken after one of the training trials. Each dot denotes the weights $\mathbf{w}_{i}=\left(\alpha_{i}, d_{i}\right)^{T}$ of a neuron.

mapping EKMs cover larger areas in the sensory input space than those in D15 or B15. Moreover, they sample distances up to $0.16 \mathrm{~m}$ whereas D15 and B15 neurons sample distances only up to $0.12 \mathrm{~m}$. Note that $0.16 \mathrm{~m}$ is the furthest that a Khepera robot can move in a single time step of 1 second. That is, indirect-mapping EKMs sample the sensory input space more completely than does D15 and B15, and thus produce finer, smoother, and more efficient motor control.

To determine whether there is statistically significant difference between the test results of different networks, $t$-tests were performed. In Table 2 , a large value indicates that the test results between two networks are similar, i.e., not significantly different (Mendenhall and Sincich, 1994). The $E$ and $T$ of indirectmapping EKMs are significantly different from those of D15 and B15 because the $t$-test values are less than 0.1. However, the differences in $E$ and $T$ of G9, 
Table 2: Significance levels from $t$-tests on similarity in performance between networks after training.

\begin{tabular}{c|ccccc}
\hline$E(\mathrm{~mm})$ & B15 & D15 & G15 & G12 & G9 \\
\hline R15 & 0.00 & 0.00 & 0.00 & 0.00 & 0.02 \\
G9 & 0.00 & 0.00 & 0.42 & 0.33 & \\
G12 & 0.01 & 0.00 & 0.21 & & \\
G15 & 0.00 & 0.00 & & & \\
D15 & 0.22 & & & & \\
\hline \multicolumn{7}{|c}{} \\
\hline$T\left(\mathrm{~m}^{-1}\right)$ & B15 & D15 & G15 & G12 & G9 \\
\hline R15 & 0.00 & 0.00 & 0.20 & 0.29 & 0.25 \\
G9 & 0.00 & 0.00 & 0.12 & 0.19 & \\
G12 & 0.00 & 0.01 & 0.47 & & \\
G15 & 0.00 & 0.01 & & & \\
D15 & 0.00 & & & & \\
\hline
\end{tabular}

\begin{tabular}{c|ccccc}
\hline$D$ & B15 & D15 & G15 & G12 & G9 \\
\hline R15 & 0.01 & 0.04 & 0.01 & 0.04 & 0.00 \\
G9 & 0.43 & 0.16 & 0.09 & 0.02 & \\
G12 & 0.04 & 0.06 & 0.09 & & \\
G15 & 0.12 & 0.09 & & & \\
D15 & 0.19 & & & & \\
\hline
\end{tabular}

G12, and G15 are not significant. This means that G9 is sufficient for the robot to stop very close to the targets at a rate that is as fast as G12 or G15. While the difference in $E$ and $D$ between R15 and indirect-mapping EKMs trained by gradient descent is significant, the difference in $T$ is not. The low $D$ of G15 is not significantly different from that of B15. The difference in $D$ of G9 from D15 and B15 is also not significant. This means that G9 achieves similar $D$ performance as D15 and B15 even though it uses less network weights.

Often, a robot is required to move through several checkpoints in a complex environment before stopping at the goal. Given that the radius of the Khepera robot is $30 \mathrm{~mm}$, it is reasonable to regard the robot to have reached (and touched) a target checkpoint if the distance-to-target $\varepsilon$ is less than $30 \mathrm{~mm}$. Figure 7 illustrates the performance comparison that evaluates this target reaching criterion after the robot has been trained.

The target reaching probability $P(\varepsilon)$ measures the probability of the robot reaching closer than a distance of $\varepsilon$ (with or without stopping) from the target locations. The normalized time-to-target $T(\varepsilon)$ measures how long it takes the robot to reach closer than a distance of $\varepsilon$ (with or without stopping) from the target locations. The mean deviation from straight-line trajectory $D(\varepsilon)$ measures how straight or wavy is the robot's trajectory.

Test results show that with indirect-mapping EKMs, the robot could get much closer to the targets with higher probability (Fig. 7A) and reach the tar- 


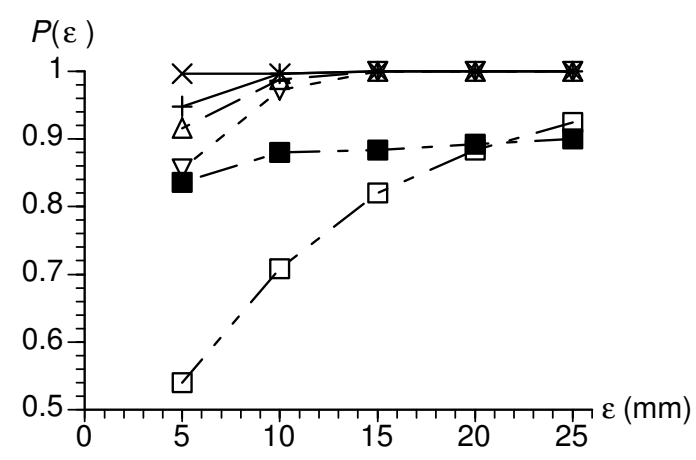

A

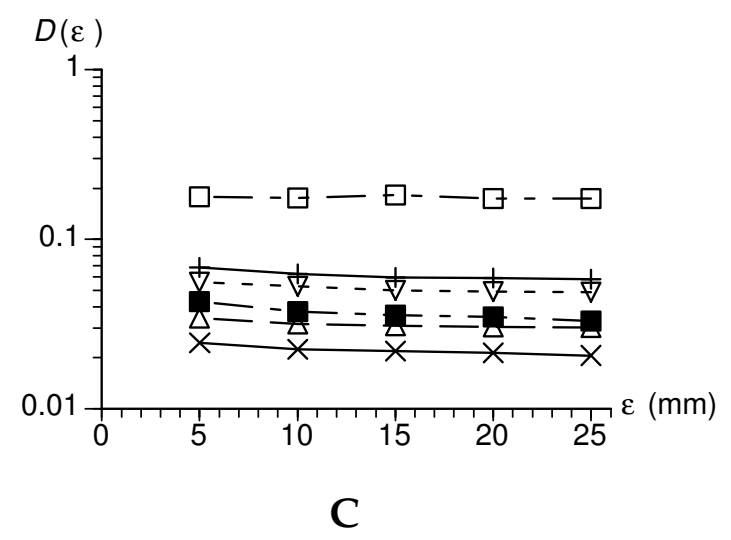

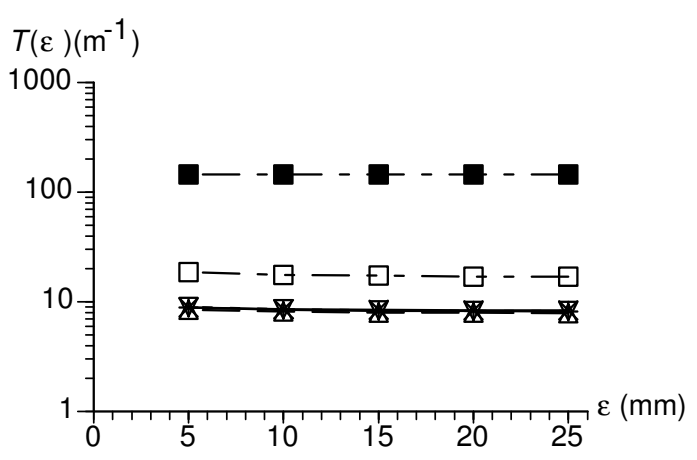

B

Figure 7: Performance comparison between various network architectures in (A) target reaching probability, (B) normalized time-to-target, and (C) mean deviation from straight line trajectory after training.

gets much faster (Fig. 7B) than with D15 or B15. Moreover, it could travel in straighter paths (Fig. 7C) than with D15.

Table 3 shows the test results for $\varepsilon=5 \mathrm{~mm}$. R15 outperformed G12 and G15 in $P(5)$, and G9, and G15 in $D(5)$. Among the indirect-mapping EKMs trained by gradient descent, G9 enabled a robot to reach closer than $5 \mathrm{~mm}$ from target locations with higher probability than G15. This could be because there were more neurons in G9 than in G15 at very small, non-zero $d$ and $|\alpha|<1.57$ radian in the input space. This set of neurons was responsible for moving the robot, at less than $10 \mathrm{~mm}$ away from the target location, forward to closer than $5 \mathrm{~mm}$. As a result, a higher $P(5)$ could be achieved. It was also observed that R15, which achieved the highest $P(5)$, had more neurons than G9 in this region of the input space. On the other hand, G15 had much more neurons at approximately zero $d$ 
Table 3: Performance comparison between networks.

\begin{tabular}{cccrc}
\hline Network & Total & \multicolumn{3}{c}{ Performance indices } \\
\cline { 3 - 5 } & parameters & $P(5)$ & \multicolumn{1}{c}{$T(5)\left(\mathrm{m}^{-1}\right)$} & $D(5)$ \\
\hline B15 & 1350 & $0.84 \pm 0.05$ & $144.70 \pm 20.16$ & $0.04 \pm 0.03$ \\
D15 & 900 & $0.54 \pm 0.07$ & $18.61 \pm 1.66$ & $0.18 \pm 0.08$ \\
G9 & 486 & $0.95 \pm 0.08$ & $8.83 \pm 0.96$ & $0.07 \pm 0.02$ \\
G12 & 864 & $0.92 \pm 0.09$ & $8.48 \pm 0.74$ & $0.03 \pm 0.02$ \\
G15 & 1350 & $0.86 \pm 0.04$ & $8.96 \pm 0.47$ & $0.06 \pm 0.01$ \\
R15 & 1350 & $1.00 \pm 0.01$ & $8.85 \pm 0.35$ & $0.03 \pm 0.01$ \\
\hline
\end{tabular}

than at very small, non-zero $d$. G12 achieved lower $D(5)$ than G9 and G15. This outcome could be justified, in a similar manner, by the explanation provided for the previous test results on $D$. By comparing the differences in normalized time-to-target and mean deviation between Tables 1 and 3, we could notice a greater amount of time and distance required for the robot to come to a stop.

One other interesting comparison is the total number of parameters or weights utilized by the various networks (Tables 1,3). G12 uses fewer weights than both D15 and B15 but still performs better comparatively.

Table 4 shows the $t$-test values at $\varepsilon=5 \mathrm{~mm}$. While the difference in $P(5)$ and $D(5)$ between R15 and indirect-mapping EKMs trained by gradient descent is significant, the difference in $T(5)$ is not. Among the indirect-mapping EKMs trained by gradient descent, the $t$-tests for $P(5)$ show no significant difference between G9 and G12, and between G12 and G15. The differences in T(5) between G9, G12, and G15 are also not significant.

To summarize, R15 achieved the best overall performance among the various networks, in particular, its performance in $E, D, P(5)$, and $D(5)$. Its $T$ and $T(5)$ performance were not significantly different from those of the other indirect-mapping EKMs. Among the indirect-mapping EKMs, it stabilized most quickly. Although B15 stabilized much faster than the other networks, it produced the poorest performance in $E, T$, and $T(5)$. D15 offered the poorest performance in $D, D(5)$ and $P(5)$. 
Table 4: Significance levels from $t$-tests on similarity in performance at $\varepsilon=5 \mathrm{~mm}$ between networks.

\begin{tabular}{c|ccccc}
\hline$P(5)$ & B15 & D15 & G15 & G12 & G9 \\
\hline R15 & 0.00 & 0.00 & 0.00 & 0.04 & 0.12 \\
G9 & 0.02 & 0.00 & 0.03 & 0.29 & \\
G12 & 0.06 & 0.00 & 0.11 & & \\
G15 & 0.26 & 0.00 & & & \\
D15 & 0.00 & & & & \\
\hline
\end{tabular}

\begin{tabular}{c|ccccc}
\hline$T(5)\left(\mathrm{m}^{-1}\right)$ & B15 & D15 & G15 & G12 & G9 \\
\hline R15 & 0.00 & 0.00 & 0.35 & 0.17 & 0.48 \\
G9 & 0.00 & 0.00 & 0.40 & 0.27 & \\
G12 & 0.00 & 0.00 & 0.13 & & \\
G15 & 0.00 & 0.00 & & & \\
D15 & 0.00 & & & & \\
\hline
\end{tabular}

\begin{tabular}{c|ccccc}
\hline$D(5)$ & B15 & D15 & G15 & G12 & G9 \\
\hline R15 & 0.12 & 0.00 & 0.00 & 0.19 & 0.00 \\
G9 & 0.09 & 0.01 & 0.14 & 0.02 & \\
G12 & 0.31 & 0.00 & 0.03 & & \\
G15 & 0.20 & 0.01 & & & \\
D15 & 0.00 & & & & \\
\hline
\end{tabular}

\subsection{Neural Network Ensemble for Target Reaching Motion with Obstacle Avoidance}

This section evaluates qualitatively and quantitatively the performance of cooperative EKMs in goal-directed, collision-free robot motion in complex, unpredictable environments. The experiments were also performed using Webots. 12 directed long-range sensors were modelled around its body of radius $3 \mathrm{~cm}$. Each sensor had a range of $17 \mathrm{~cm}$, enabling the detection of obstacles at $20 \mathrm{~cm}$ or nearer from the robot's center, and a resolution of $0.5 \mathrm{~cm}$ to simulate noise.

Two tests were performed to compare cooperative EKMs with another ensemble method (Low et al., 2002). The latter approach, termed command fusion, linearly combines the motion control outputs, using weighted sum, of different neural networks implementing different behaviors. This is a widely used technique to integrate the motion control outputs produced by different neural networks (Hashem, 1997; Haykin, 1999; Jacobs, 1995). For our case, the target reaching motion is produced by an indirect-mapping EKM while obstacle avoidance is performed using the method of Braitenberg's Type-3C vehicle (Braitenberg, 1984). To elaborate, when the robot senses the presence of an obstacle, say, in front and on the left, the right motor will rotate backward faster than the left motor's rotation forward, thus turning the robot away from the 


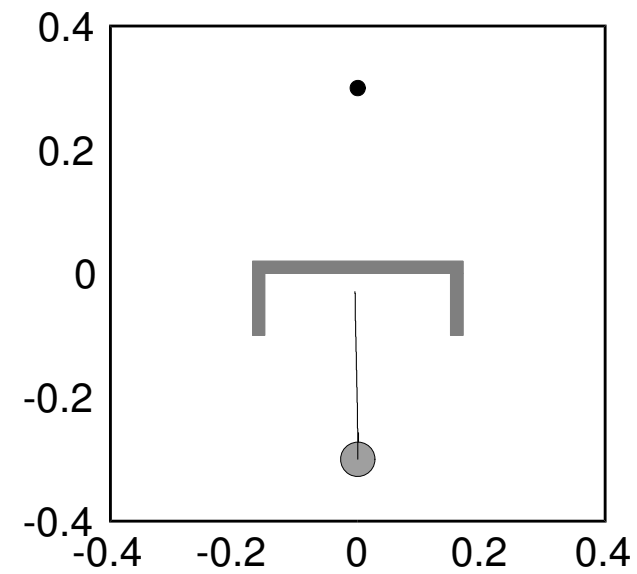

A

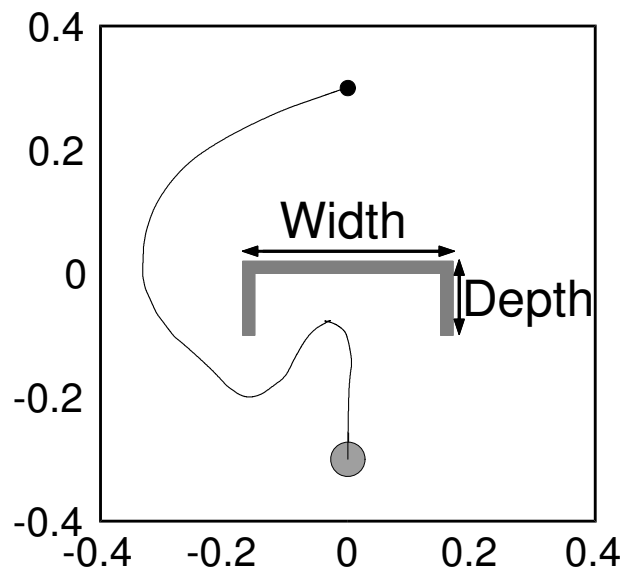

B

Figure 8: Negotiating unforeseen concave obstacle that was $34 \mathrm{~cm}$ wide and $12 \mathrm{~cm}$ deep. (A) The robot using command fusion was trapped but (B) the one adopting cooperative EKMs successfully moved around the obstacle.

obstacle.

For both ensemble methods, the target reaching and obstacle avoidance modules ran at intervals of $256 \mathrm{~ms}$ and $128 \mathrm{~ms}$ respectively. The robot's performance was assessed in an environment under two unforeseen conditions: (1) concave obstacle, and (2) narrow doorway between closely spaced obstacles.

In the first test (Fig. 8), the robot fitted with command fusion got trapped by the concave obstacle (Fig. 8A). The target reaching behavior tried to move the robot forward to reach the target while the obstacle avoidance behavior moved it backward to avoid the obstacle. The combined output cancelled each other, causing the robot to be trapped by the obstacle. On the other hand, the robot with cooperative EKMs could overcome the obstacle to reach the goal successfully (Fig. 8B).

It is noted that a robot with cooperative EKMs can still get trapped if the obstacle is so concave that the obstacle fields cannot completely inhibit the neurons at or near the target location. Figure 9 shows the maximum obstacle width that a robot with cooperative EKMs can overcome with varying obstacle depths and robot sensing ranges. Given a fixed sensing range, the maximum negotiable obstacle depth decreases with increasing width. When the sensing range increases, the robot with cooperative EKMs can negotiate extremely wide concave obstacle if it is not too deep. Conversely, to be able to overcome a fairly 


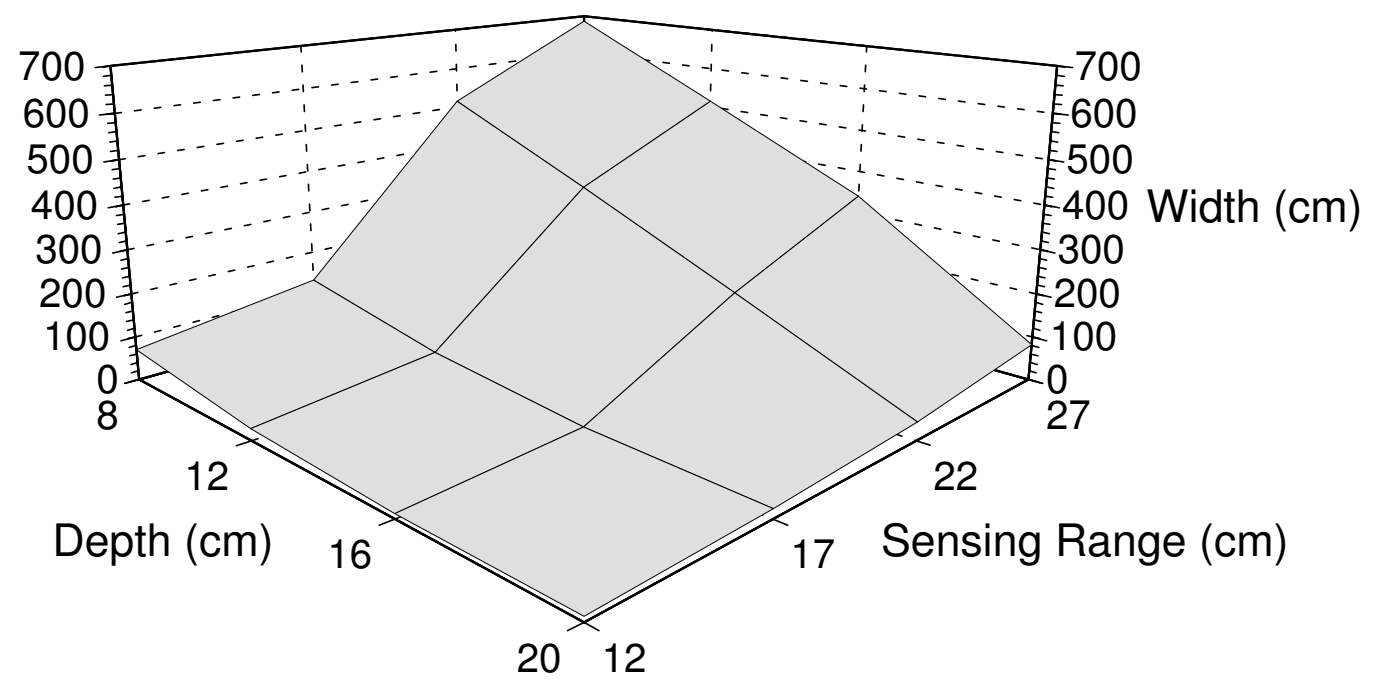

Figure 9: Maximum width of concave obstacle (Fig. 8B) that a robot with cooperative EKMs can overcome with different combinations of obstacle depths and robot sensing ranges.

deep obstacle, its width cannot be too large.

The abovementioned limitation, however, does not diminish the significance of our method as it is simpler than many existing reactive robot motion methods for overcoming unforeseen concave obstacles (Lagoudakis and Maida, 1999; Liu et al., 2000; Zelek and Levine, 1996). In particular, it utilizes only local information of the target location and the unforeseen obstacles, as opposed to motion planners (Latombe, 1999) that require global knowledge of the environment to operate.

In the second test (Fig. 10), the robot endowed with command fusion could not pass through the narrow doorway between closely spaced obstacles (Fig. 10A) because its obstacle avoidance behavior counteracted the target reaching behavior. In contrast, the robot with cooperative EKMs could always traverse through the narrow doorway to the goal successfully (Fig. 10B).

These two simple tests show that for command fusion, though each neural network proposes an action that is optimal by itself, the weighted sum of these action commands produces a combined action that may not satisfy the overall task. Cooperative EKMs, however, considers the activity signals of each localization EKM and integrates them to determine an action that can satisfy each localization EKM to a certain degree. Such tightly coupled interaction between the localization EKMs and the motor control EKM in the cooperative EKMs 


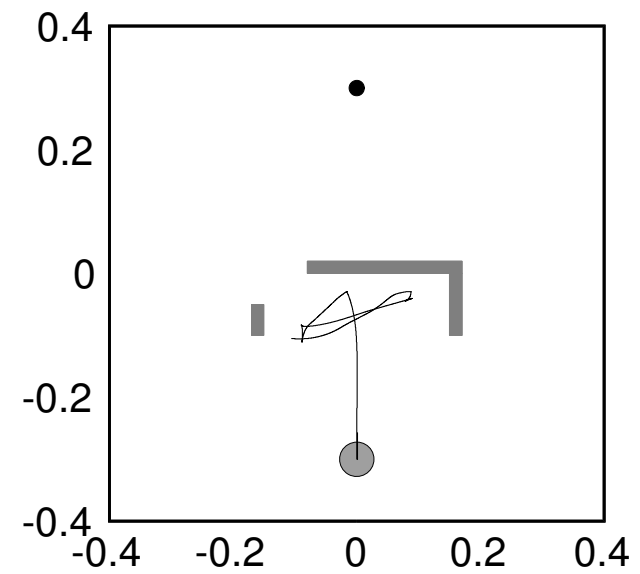

A

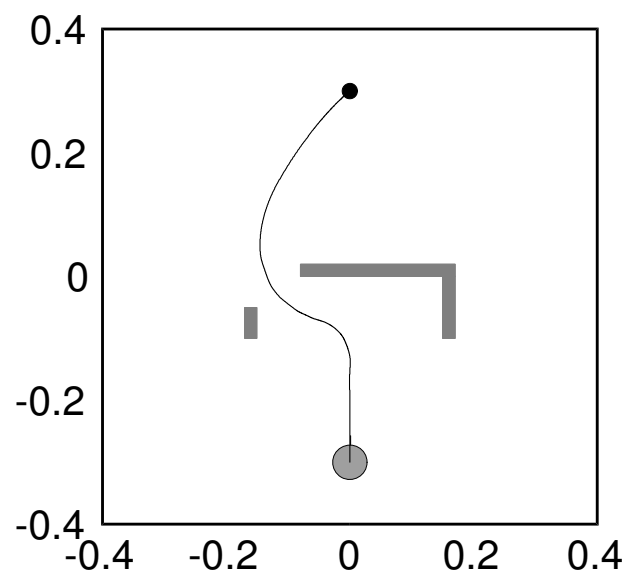

B

Figure 10: Passing through unforeseen narrow doorway between closely spaced obstacles that was $86 \mathrm{~mm}$ wide. (A) The robot using command fusion was trapped but (B) the one adopting cooperative EKMs successfully passed through the narrow doorway to the goal.

framework enables the robot to achieve more complex tasks.

Recall that the standard deviations $\sigma$ of the Gaussian functions for the target and obstacle fields play an important role in the robot motion capabilities of cooperative EKMs (Sections 3.2 and 3.3). For the target field (Fig. 3A), $\sigma_{a \alpha}$ and $\sigma_{a d}$ control the elongation of the target field perpendicular to and along the target direction respectively. Parameters $\sigma_{b \alpha}$ and $\sigma_{b d}$ achieve a similar effect for the obstacle field. For the negotiation of concave obstacles (e.g., Fig. 8B), the target field has to be considerably elongated perpendicular to the target direction. This requires a large enough $\sigma_{a \alpha}$ parameter value. However, as this value increases, the tendency of the robot moving along the shorter path to the goal via the narrow doorway (Fig. 10B) decreases and the longer detour featured in Fig. 8B is increasingly preferred in the situation of Fig. 10B. When $\sigma_{a \alpha}$ is large, subtraction of the obstacle field from the highly elongated target field (Fig. 3D) in the motor control EKM may result in the neuron at the edge of the concave obstacle to be most highly activated, rather than the neuron at the narrow doorway. Nonetheless, the above two tests and the subsequent ones can be achieved by a single $\sigma_{a \alpha}$ value of 2.475. If $\sigma_{a d}$ is too small, the target field may be totally suppressed by the obstacle field depending on $\sigma_{a \alpha}$. This may or may not cause the robot to be trapped in the concave obstacle since any 
neuron not inhibited by the obstacle field can be potentially activated. If $\sigma_{a d}$ is too large, the robot may get trapped in the concave obstacle. Superposition of the fields may cause the neuron in the cavity of the concave obstacle to be most highly activated, rather than the neuron at the edge of the obstacle. In all the tests, $\sigma_{a d}$ is set to 0.0495 .

The parameter values of $\sigma_{b \alpha}$ and $\sigma_{b d}$ have to be large enough for the robot to avoid collision with obstacles as well as discriminate whether a doorway is wide enough to pass through. However, if these values are too large, the robot cannot move to target locations near the obstacles nor detect the presence of narrow but traversable doorways. In all the tests, $\sigma_{b \alpha}$ is set to 0.495 while $\sigma_{b d}$ uses the values given in Eq. 8. The above evaluation of the target and obstacle field parameters highlights their significance to the robot motion capabilities of cooperative EKMs. In our future work, we will consider using reinforcement learning to train the appropriate parameter values for negotiating different obstacles when the robot encounters them during motion.

The next two tests aim to demonstrate the capabilities of cooperative EKMs in performing more complex motion tasks. The environment for the first test consisted of three rooms connected by two doorways (Fig. 11). The middle room contained two obstacles moving in anticlockwise circular paths. The robot began in the left-most room and was tasked to move to the right-most room. Test results show that the robot was able to negotiate past the extended walls and the dynamic obstacles to reach the goal. Note that this target reaching motion was completely determined by the cooperation and competition between the EKMs and no global planning was used.

The environment for the second test consisted of three rooms connected by two doorways and some unforeseen static obstacles (Fig. 12). The robot began in the top corner of the left-most room and was tasked to move into the narrow corner of the right-most room via checkpoints plotted by a planner (Low et al., 2002). The robot was able to move through the checkpoints to the goal by traversing between narrowly spaced convex obstacles in the first and the last room, and overcoming an unforeseen concave obstacle in the middle room. The results of these last two tests further confirm the effectiveness of cooperative EKMs in handling complex tasks in complex, unpredictable environments.

\section{Conclusion}

This paper presents a new approach of learning sensorimotor control for complex robot motion tasks using cooperative EKMs. Quantitative evaluation re- 

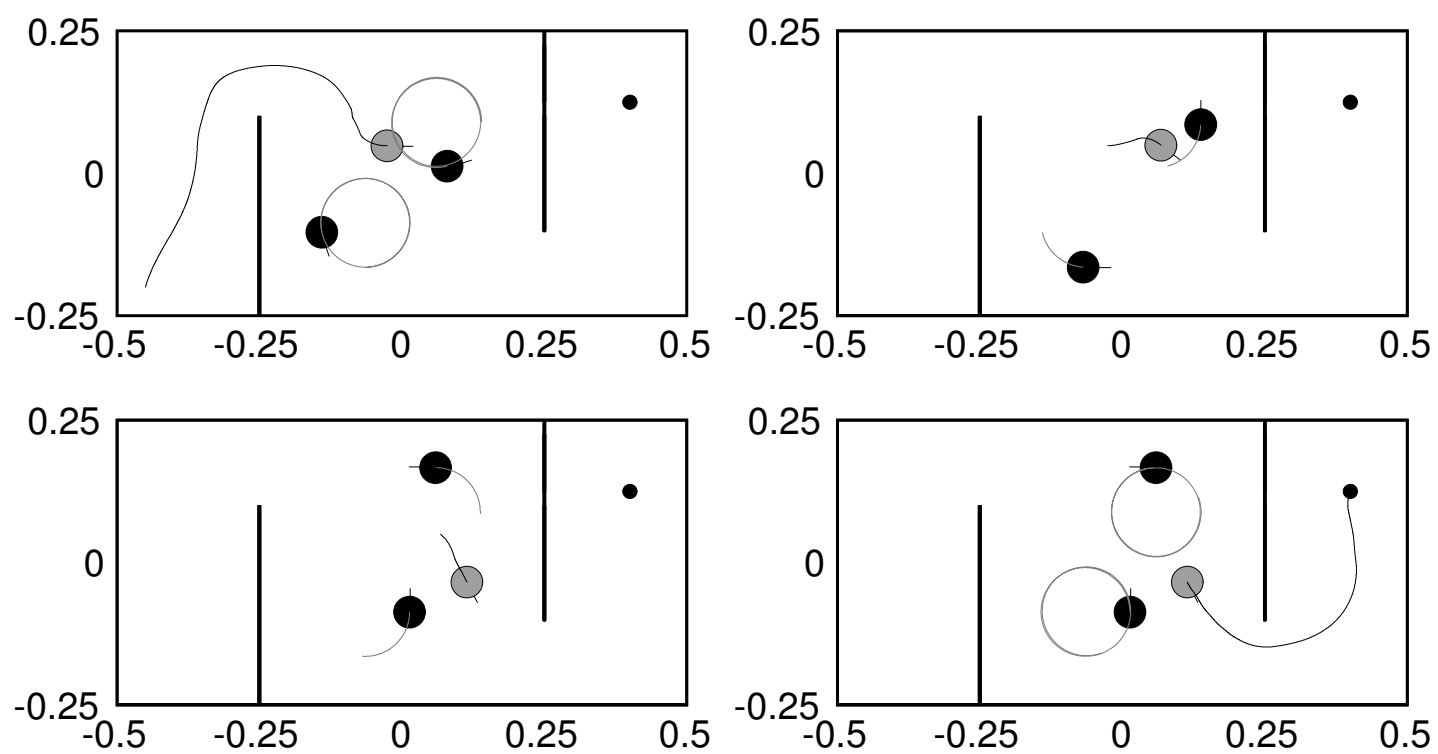

Figure 11: Motion of robot (gray) in an environment with two unforeseen obstacles (black) moving in anticlockwise circular paths. The robot could successfully negotiate past the extended walls and the dynamic obstacles to reach the goal (small black dot).

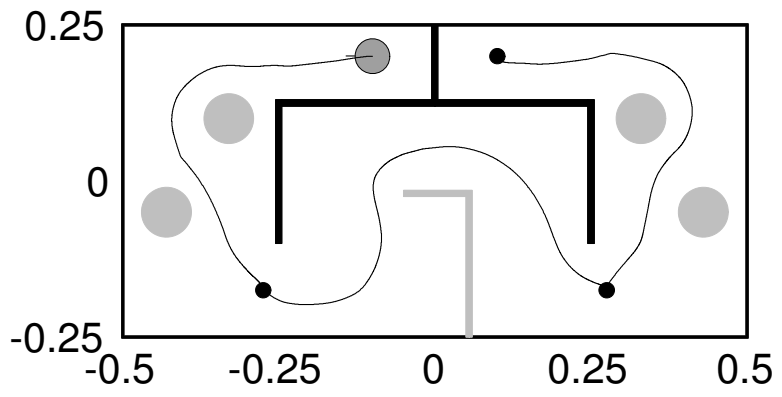

Figure 12: Motion of robot (dark gray) in a complex environment. The checkpoints (small black dots) were located at the doorways and the goal position. The robot could successfully navigate through the checkpoints to the goal by traversing between unforeseen narrowly spaced convex obstacles (light gray) in the first and the last room and overcoming an unforeseen concave obstacle (light gray) in the middle room. 
veals that indirect-mapping EKM can produce finer, smoother, and more efficient robot motion control than other local learning methods such as directmapping EKM and BFN. Furthermore, training the control parameters of the indirect-mapping EKM with recursive least squares allows faster convergence and more superior performance than with gradient descent. The cooperation and competition of multiple EKMs enable the non-holonomic mobile robot to negotiate unforeseen concave, closely spaced, and dynamic obstacles. These tasks can easily trap robots that are controlled by neural network ensembles employing command fusion techniques. Cooperative EKMs can thus augment the reactive capabilities of an autonomous mobile robot significantly. Recently, we have enhanced cooperative EKMs further to achieve multi-robot motion tasks such that multiple robots fitted with cooperative EKMs can coordinate their tracking of moving targets (Fig. 13). Qualitative and quantitative test results of the improved cooperative EKMs for multi-robot tasks are presented in (Low et al., 2003, 2004). Our continuing research goal is to generalize this approach to other sensorimotor control problems such as those of static and mobile robot manipulators.

\section{References}

Arkin, R. C. (1998). Behavior-Based Robotics. MIT Press, Cambridge, MA.

Atkeson, C. G., Moore, A. W., and Schaal, S. (1997). Locally weighted learning. Artificial Intelligence Review, 11(1-5), 11-73.

Baird, L. C. (1995). Residual algorithms: Reinforcement learning with function approximation. In Proc. 12th International Conference on Machine Learning (ICML-95), pages 30-37.

Battiti, R. (1992). First and second-order methods for learning: Between steepest descent and Newton's method. Neural Comput., 4(2), 141-166.

Battiti, R. and Colla, A. (1994). Democracy in neural nets: Voting schemes for classification. Neural Networks, 7(4), 691-707.

Beer, R. D. (1995). A dynamical systems perspective on agent-environment interaction. Artificial Intelligence, 72(1-2), 173-215.

Braitenberg, V. (1984). Vehicles: Experiments in Synthetic Psychology. MIT Press. 


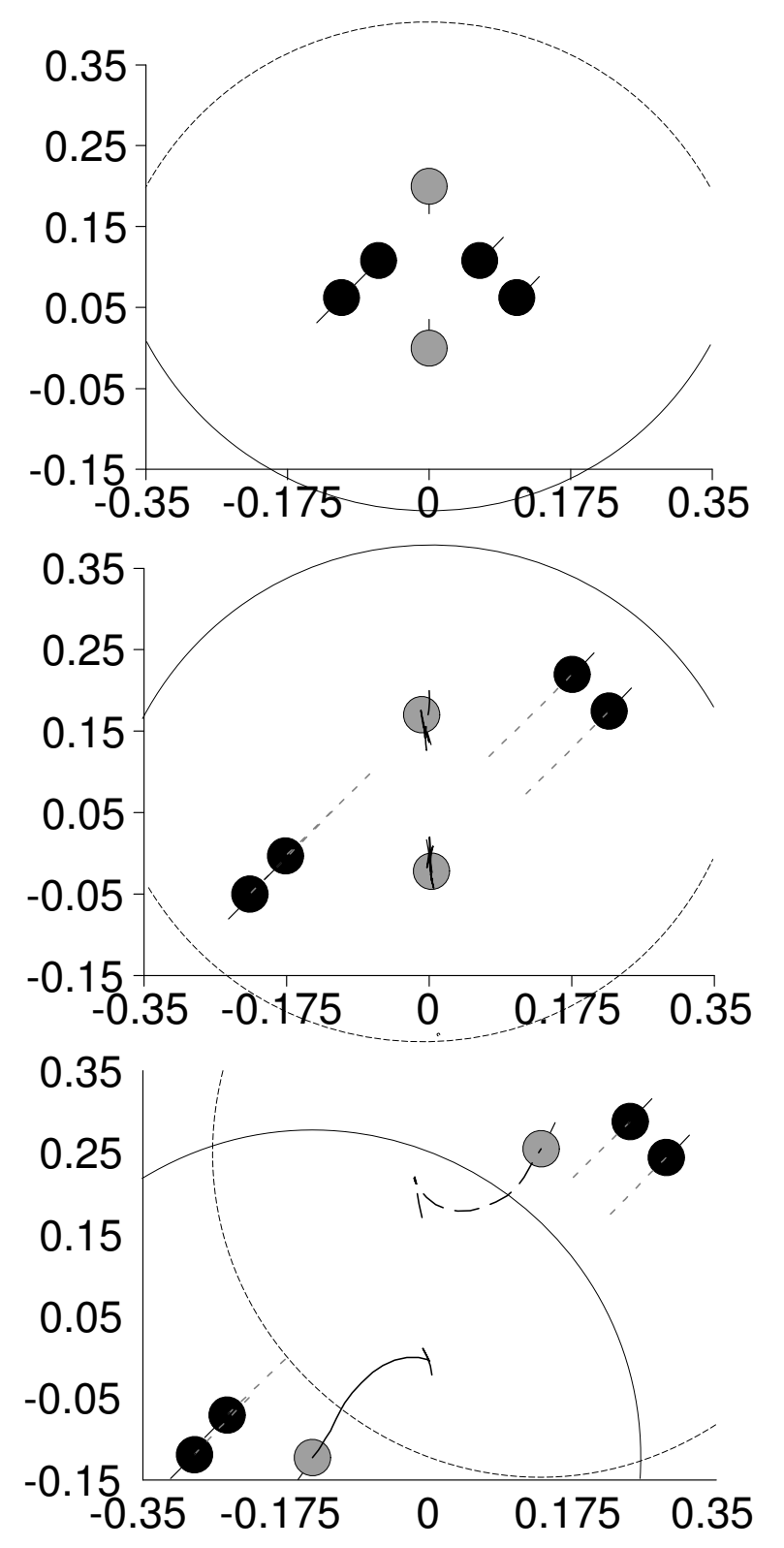

Figure 13: Cooperative tracking of moving targets. When the targets were moving out of the robots' sensory range, the two robots moved in opposite directions to track the targets. In this way, all targets could still be observed by the robots. 
Bruske, J. and Sommer, G. (1995). Dynamic cell structure learns perfectly topology preserving map. Neural Comput., 7(4), 845-865.

Burgard, W., Cremers, A. B., Fox, D., Hähnel, D., Lakemeyer, G., Schulz, D., Steiner, W., and Thrun, S. (1999). Experiences with an interactive museum tour-guide robot. Artificial Intelligence, 114(1-2), 3-55.

Cameron, S., Grossberg, S., and Guenther, F. H. (1998). A self-organizing neural network architecture for navigation using optic flow. Neural Comput., 10(2), 313-352.

Davids, A. (2002). Urban search and rescue robots: From tragedy to technology. IEEE Intelligent Systems, 17(2), 81-83.

Decugis, V. and Ferber, J. (1998). Action selection in an autonomous agent with a hierarchical distributed reactive planning architecture. In Proc. 2 nd International Conference on Autonomous Agents, pages 354-361.

Dietterich, T. G. (2000). Hierarchical reinforcement learning with the MAXQ value function decomposition. J. Artificial Intelligence Res., 13, 227-303.

Fiorini, P., Kawamura, K., and Prassler, E., editors. (2000). Autonomous Robots, Special Issue on Cleaning and Housekeeping Robots, 9(3).

Ghosh, J. and Nag, A. (2001). An overview of radial basis function networks. In R. J. Howlett and L. C. Jain, editors, Radial Basis Function Networks 2: New Advances in Design, pages 1-36. Physica-Verlag, New York.

Glentis, G.-O., Berberidis, K., and Theodoridis, S. (1999). Efficient least squares adaptive algorithms for FIR transversal filtering. IEEE Signal Processing Mag., 16(4), 13-41.

Gorinevsky, D. and Connolly, T. H. (1994). Comparison of some neural networks and scattered data approximations: The inverse manipulator kinematics example. Neural Comput., 6(3), 521-542.

Gross, H.-M., Stephan, V., and Krabbes, M. (1998). A neural field approach to topological reinforcement learning in continuous action spaces. In Proc. International Joint Conference on Neural Networks, volume 3, pages 1992-1997.

Hansen, L. K. and Salamon, P. (1990). Neural network ensembles. IEEE Trans. Pattern Anal. Machine Intell., 12(10), 993-1001. 
Hartman, E. and Keeler, D. (1991). Predicting the future: Advantages of semilocal units. Neural Comput., 3(4), 566-578.

Hashem, S. (1997). Optimal linear combinations of neural networks. Neural Networks, 10(4), 599-614.

Hayes, A. T., Martinoli, A., and Goodman, R. M. (2002). Distributed odor source localization. IEEE Sensors, 2(3), 260-271.

Haykin, S. (1999). Neural Networks: A Comprehensive Foundation. Prentice Hall, Upper Saddle River, NJ, second edition.

Haykin, S. (2002). Adaptive Filter Theory. Prentice Hall, Upper Saddle River, NJ, fourth edition.

Heikkonen, J. and Koikkalainen, P. (1997). Self-organization and autonomous robots. In O. Omidvar and P. van der Smagt, editors, Neural Systems for Robotics, pages 297-337. Academic Press.

Hertzberg, J., Christaller, T., Kirchner, F., Licht, U., and Rome, E. (1998). Sewer robotics. In R. Pfeifer, B. Blumberg, J.-A. Meyer, and S. W. Wilson, editors, From Animals to Animats 5: Proc. International Conference on Simulation of Adaptive Behavior, pages 427-436. MIT Press: Cambridge, MA.

Howard, A., Matarić, M. J., and Sukhatme, G. S. (2002). Network deployment using potential fields: A distributed, scalable solution to the area coverage problem. In H. Asama, T. Arai, T. Fukuda, and T. Hasegawa, editors, Distributed Autonomous Robotic Systems 5: Proc. 6th International Symposium on Distributed Autonomous Robotic Systems, pages 299-308. Springer: New York.

Huntsberger, T. and Rose, J. (1998). BISMARC: A biologically inspired system for map-based autonomous rover control. Neural Networks, 11(7-8), 14971510.

Ijspeert, A. J., Martinoli, A., Billard, A., and Gambardella, L. M. (2001). Collaboration through the exploitation of local interactions in autonomous collective robotics: The stick pulling experiment. Autonomous Robots, 11(2), 149-171.

Jacobs, R. A. (1995). Methods for combining experts' probability assessments. Neural Comput., 7(5), 867-888.

Jansen, A., van der Smagt, P., and Groen, F. C. A. (1995). Nested networks for robot control. In A. F. Murray, editor, Applications of Neural Networks, pages 221-239. Kluwer Academic Publishers, Dordrecht, the Netherlands. 
Kaelbling, L. P., Littman, M. L., and Moore, A. W. (1996). Reinforcement learning: A survey. J. Artificial Intelligence Res., 4, 237-285.

Karayiannis, N. B. (1999). Reformulated radial basis neural networks trained by gradient descent. IEEE Trans. Neural Networks, 10(3), 657-671.

Karayiannis, N. B. and Mi, G. W. (1997). Growing radial basis neural networks: Merging supervised and unsupervised learning with network growth techniques. IEEE Trans. Neural Networks, 8(6), 1492-1506.

Kim, J.-O. and Khosla, P. (1992). Real-time obstacle avoidance using harmonic potential functions. IEEE Trans. Robot. Automat., 8(3), 338-349.

Kittler, J., Hatef, M., Duin, R. P. W., and Matas, J. (1998). On combining classifiers. IEEE Trans. Pattern Anal. Machine Intell., 20(3), 226-239.

Kohonen, T. (2000). Self-Organizing Maps. Springer, New York, third edition.

Koren, Y. and Borenstein, J. (1991). Potential field methods and their inherent limitations for mobile robot navigation. In Proc. IEEE International Conference on Robotics and Automation (ICRA'91), pages 1394-1404.

Kuperstein, M. (1991). INFANT neural controller for adaptive sensory-motor coordination. Neural Networks, 4(2), 131-146.

Lagoudakis, M. G. and Maida, A. S. (1999). Robot navigation with a polar neural map, Student Abstract. In Proc. 16th National Conference on Artificial Intelligence (AAAI-99), page 965.

Latombe, J.-C. (1999). Motion planning: A journey of robots, molecules, digital actors, and other artifacts. International Journal of Robotics Research, 18(11), 1119-1128.

Littmann, E. and Ritter, H. (1996). Learning and generalization in cascade network architectures. Neural Comput., 8(7), 1521-1539.

Liu, C., Ang Jr., M. H., Krishnan, H., and Lim, S. Y. (2000). Virtual obstacle concept for local-minimum-recovery in potential-field based navigation. In Proc. IEEE International Conference on Robotics and Automation (ICRA'00), volume 2, pages 983-988.

Low, K. H., Leow, W. K., and Ang, Jr., M. H. (2002). A hybrid mobile robot architecture with integrated planning and control. In Proc. 1st International 
Joint Conference on Autonomous Agents and MultiAgent Systems (AAMAS-02), pages 219-226.

Low, K. H., Leow, W. K., and Ang, Jr., M. H. (2003). Action selection for singleand multi-robot tasks using cooperative extended Kohonen maps. In Proc. 18th International Joint Conference on Artificial Intelligence (IJCAI-03), pages 1505-1506.

Low, K. H., Leow, W. K., and Ang, Jr., M. H. (2004). Task allocation via selforganizing swarm coalitions in distributed mobile sensor network. In Proc. 19th National Conference on Artificial Intelligence (AAAI-04), pages 28-33.

Maes, P. (1995). Modeling adaptive autonomous agents. In C. G. Langton, editor, Artificial Life : An Overview, pages 135-162. MIT Press: Cambridge, MA. Also appeared in Artificial Life, 1(1-2).

Mahadevan, S. and Connell, J. (1992). Automatic programming of behaviorbased robots using reinforcement learning. Artificial Intelligence, 55(2-3), 311365 .

Martinetz, T. M., Ritter, H. J., and Schulten, K. J. (1990). Three-dimensional neural net for learning visuomotor coordination of a robot arm. IEEE Trans. Neural Networks, 1(1), 131-136.

Martinoli, A., Ijspeert, A. J., and Mondada, F. (1999). Understanding collective aggregation mechanisms: From probabilistic modelling to experiments with real robots. Robotics and Autonomous Systems, 29(1), 51-63.

Mendenhall, W. and Sincich, T. (1994). Statistics for Engineering and the Sciences. Prentice Hall, fourth edition.

Michel, O. (2004). Cyberbotics Ltd. WebotsTM: Professional mobile robot simulation. International Journal of Advanced Robotic Systems, 1(1), 39-42.

Millán, J. del R., Posenato, D., and Dedieu, E. (2002). Continuous-action Qlearning. Machine Learning, 49(2-3), 249-265.

Moody, J. and Darken, C. J. (1989). Fast learning in networks of locally-tuned processing units. Neural Comput., 1(2), 281-294.

Platt, J. (1991). A resource-allocating network for function interpolation. Neural Comput., 3(2), 213-225. 
Poggio, T. and Girosi, F. (1990). Networks for approximation and learning. Proc. IEEE, 78(9), 1481-1497.

Pomerleau, D. A. (1991). Efficient training of artificial neural networks for autonomous navigation. Neural Comput., 3(1), 88-97.

Port, R. F. and van Gelder, T. (1995). Mind as Motion: Explorations in the Dynamics of Cognition. MIT Press, Cambridge, MA.

Rao, R. P. H. and Fuentes, O. (1998). Hierarchical learning of navigational behaviors in an autonomous robot using a predictive sparse distributed memory. Machine Learning, 31(1-3), 87-113.

Rimon, E. and Koditschek, D. E. (1992). Exact robot navigation using artificial potential functions. IEEE Trans. Robot. Automat., 8(5), 501-518.

Ritter, H. and Schulten, K. (1986). Topology conserving mappings for learning motor tasks. In J. S. Denker, editor, Neural Networks for Computing, pages 376380. American Institute of Physics Publication, Conference Proceedings 151, Snowbird, Utah.

Rohanimanesh, K. and Mahadevan, S. (2003). Learning to take concurrent actions. In S. Becker, S. Thrun, and K. Obermayer, editors, Advances in Neural Information Processing Systems 15, pages 1619-1626, Cambridge, MA. MIT Press.

Rosenblatt, J. K. (1997). DAMN: A distributed architecture for mobile navigation. J. Expt. Theor. Artif. Intell., 9(2-3), 339-360.

Russell, S. and Norvig, P. (1995). Artificial Intelligence: A Modern Approach. Prentice Hall, Upper Saddle River, NJ.

Rybski, P. E., Stoeter, S. A., Gini, M., Hougen, D. F., and Papanikolopoulos, N. P. (2002). Performance of a distributed robotic system using shared communications channels. IEEE Trans. Robot. Automat., 18(5), 713-727.

Santamária, J. C., Sutton, R., and Ram, A. (1998). Experiments with reinforcement learning in problems with continuous state and action spaces. Adaptive Behavior, 6(2), 163-218.

Schaal, S. and Atkeson, C. G. (1998). Constructive incremental learning from only local information. Neural Comput., 10(8), 2047-2084. 
Schaal, S., Atkeson, C. G., and Vijayakumar, S. (2002). Scalable techniques from nonparametric statistics for real time robot learning. Applied Intelligence, 17(1), 49-60.

Sharkey, A. J. C. and Sharkey, N. E. (1997). Combining diverse neural nets. Knowledge Engineering Review, 12(3), 231-247.

Sharkey, N. E. (1998). Learning from innate behaviors: A quantitative evaluation of neural network controllers. Machine Learning, 31(1-3), 115-139. Also appeared in Autonomous Robots, vol. 5, no. 3-4, pp. 317-334.

Shastri, S. V., editor. (1999). International Journal of Robotics Research, Special Issue on Field and Service Robotics, 18(7).

Smart, W. D. and Kaelbling, L. P. (2000). Practical reinforcement learning in continuous spaces. In Proc. 17th International Conference on Machine Learning (ICML-00), pages 903-910.

Smith, A. J. (2002). Applications of the self-organising map to reinforcement learning. Neural Networks, 15(8-9), 1107-1124.

Sutton, R. (1998). Reinforcement Learning: An Introduction. MIT Press, Cambridge, MA.

Tani, J. and Fukumura, N. (1994). Learning goal-directed sensory-based navigation of a mobile robot. Neural Networks, 7(3), 553-563.

Touzet, C. (1997). Neural reinforcement learning for behavior synthesis. Robotics and Autonomous Systems, 22(3-4), 251-281.

van der Smagt, P., Groen, F. C. A., and van het Groenewoud, F. (1994). The locally linear nested network for robot manipulation. In Proc. International Conference on Neural Networks, volume 5, pages 2787-2792.

Versino, C. and Gambardella, L. M. (1995). Learning the visuomotor coordination of a mobile robot by using the invertible Kohonen map. In J. Mira and F. Sandoval, editors, Proc. International Workshop on Artificial Neural Networks, pages 1084-1091. LNCS 930, Springer, Berlin.

Walter, J. and Ritter, H. (1996). Investment learning with hierarchical PSOM. In D. Touretzky, M. Mozer, and M. Hasselmo, editors, Advances in Neural Information Processing Systems 8, pages 570-576, Cambridge, MA. MIT Press. 
Walter, J. A. and Schulten, K. J. (1993). Implementation of self-organizing neural networks for visuo-motor control of an industrial robot. IEEE Trans. Neural Networks, 4(1), 86-95.

Wettschereck, D. and Dietterich, T. (1992). Improving the performance of radial basis function networks by learning center locations. In J. E. Moody, S. J. Hanson, and R. P. Lipmann, editors, Advances in Neural Information Processing Systems 4, pages 1133-1140, Morgan Kaufmann: San Mateo, CA.

Zalama, E., Gaudiano, P., and Coronado, J. L. (1995). A real-time, unsupervised neural network for the low-level control of a mobile robot in a non-stationary environment. Neural Networks, 8(1), 103-123.

Zelek, J. S. and Levine, M. D. (1996). SPOTT: A mobile robot control architecture for unknown or partially known environments. In I. Nourbakhsh, editor, Planning with Incomplete Information for Robot Problems: Papers from the AAAI Spring Symposium, pages 129-140. AAAI Press. 